\title{
On Certain Infinite Dimensional Aspects of Arakelov Intersection Theory
}

\author{
Jean-Michel Bismut \\ Département de Mathématique, Université Paris-Sud, F-91405 Orsay, France
}

Received June 14, 1991

\begin{abstract}
Let $k: Y \rightarrow X$ be an embedding of compact complex manifolds. Bismut and Lebeau have calculated the Quillen norm of the canonical isomorphism identifying the determinant of the cohomology of a holomorphic vector bundle over $Y$ and the determinant of the cohomology of a resolution by a complex of holomorphic vector bundles over $X$. The purpose of this paper is to show that the formula of BismutLebeau can be viewed as an equivariant intersection formula over the loop space of the considered manifolds, in the presence of an infinite dimensional excess normal bundle. This excess normal bundle is responsible for the appearance of the additive genus $R$ of Gillet and Soulé in the formula of Bismut and Lebeau.
\end{abstract}

\section{Table of Contents}

I. Bott-Chern Currents in Finite Dimensions and Euler-Green Currents in Infinite Dimensions . . . . . . . . . . . . . . . . . . . . . . . . . . . . . . 221

a) Complex Embeddings and Loop Spaces . . . . . . . . . . . . . . . . . . 221

b) Accumulating Evidence: Some Finite Dimensional Formulas . . . . . . . . . . 223

II. Quillen Metrics and Resolutions _ . . . . . . . . . . . . . . . . . . . . 227

a) The Quillen Metric on the Inverse of the Determinant of the Cohomology . . . . 228

b) Quillen Metrics and Resolutions . . . . . . . . . . . . . . . . . . . . . 229

III. Equivariant Intersection Over Loop Spaces and a Formula of Bismut-Lebeau . . 230

a) An Equivariantly Closed Differential form on $L X$. . . . . . . . . . . . . . 230

b) Accumulating Evidence: A Formal Expression for $\frac{\partial \theta^{\xi}}{\partial s}(0)$. . . . . . . . . . 231

c) Accumulating Evidence: A Formal Expression for the Ray-Singer Analytic Torsion of $\Lambda E^{*} \otimes \eta$. . . . . . . . . . . . . . . . . . . . . . . . . . . . . . . . . . . 233

d) Accumulating Evidence: A very Formal Expression for $\log \left(|\sigma|_{\lambda^{-1}\left(\eta_{\mid Y}\right) \otimes \lambda\left(\Lambda E^{*} \otimes \eta\right)}^{2}\right) \quad 235$

e) Accumulating Evidence: A Formal Expression for $\log \left(\|\sigma\|_{\lambda^{-1}\left(\eta_{\mid Y}\right) \otimes \lambda\left(\Lambda E^{*} \otimes \eta\right)}^{2}\right) \quad$. . 238

f) A Formal Expression for Certain Finite Dimensional Integrals . . . . . . . . . 239

g) A Formal Formula $\log \left(\|\sigma\|_{\lambda^{-1}\left(\eta_{\mid Y}\right) \otimes \lambda\left(\Lambda E^{*} \otimes \eta\right)}^{2}\right)$ in Terms of Finite Dimensional Integrals . . . . . . . . . $\left(\eta_{\mid Y}\right) \otimes \lambda\left(\Lambda E^{*} \otimes \eta\right)$. . . . . . . . . . . . . . . 242

h) A theorem of Bismut-Lebeau . . . . . . . . . . . . . . . . . . . . 243

i) One Word of Explanation . . . . . . . . . . . . . . . . . . . . . . . . . . 244

References . . . . . . . . . . . . . . . . . . . . . . 247 
The purpose of this paper is to exhibit certain remarkable relations of Quillen metrics on the determinant of the cohomology to equivariant Arakelov theory on certain infinite dimensional manifolds. In particular, we will show that the result of Bismut and Lebeau [BL1,2] describing the behaviour of Quillen metrics under complex embeddings can be viewed as a formal consequence of an intersection formula on the loop space of the considered manifold, in the presence of an infinite dimensional excess normal bundle. This intersection formula was first established in a finite dimensional context in a previous paper [B2].

Let us briefly recall the formalism of [B2]. Let $\left(L X, \omega^{L X}\right)$ be a compact Kähler manifold, with Kähler form $\omega^{L X}$. Let $K$ be a holomorphic Killing vector field on $L X$.

Set

$$
\begin{gathered}
\partial_{K}=\partial+i_{K^{(0,1)}}, \\
\bar{\partial}_{K}=\bar{\partial}+i_{K^{(1,0)}}, \\
X=\{x \in L X ; K(x)=0\} .
\end{gathered}
$$

Let $N_{X / L X}$ be the normal bundle to $X$ in $L X$. Then $N_{X / L X}$ is naturally equipped with a metric $g^{N_{X / L X}}$. Let ${ }^{K} c_{\max }\left(N_{X / L X}, g^{N_{X / L X}}\right)$ be the Chern-Weil representative of the equivariant Euler class of $N_{X / L X}$ associated to the holomorphic Hermitian connection on $\left(N_{X / L X}, g^{N_{X / L X}}\right)$.

In [B8], we constructed a $K$-invariant current ${ }^{K} S_{\omega L X}$ on $L X$, whose wave front set is included in $N_{L Y / L X, \mathbb{R}}^{*}$, and is such that

$$
\frac{\bar{\partial}_{K} \partial_{K}}{2 i \pi}{ }^{K} S_{\omega L X}=1-{ }^{K} c_{\max }^{-1}\left(N_{X / L X}, g^{N_{X / L X}}\right) \delta_{X} .
$$

Equation (0.2) refines on the localization formulas in equivariant cohomology of Berline-Vergne [BeV], which themselves extend related formulas of Bott [Bo].

Let $\left(L E, g^{L E}\right)$ be a $K$-equivariant holomorphic Hermitian vector bundle over $L X$ and let $r$ be a $K$-invariant holomorphic section of $L E$. Set

$$
L Y=\{x \in L X ; r(x)=0\}
$$

Assume that $L Y$ is a complex submanifold of $L X$. In [B2], we constructed a $K$ invariant current $K^{K} \tilde{e}^{L X}\left(L E, g^{L E}\right)$, whose wave front set is included in $N_{L Y / L X, \mathbb{R}}^{*}$, such that

$$
\frac{\bar{\partial}_{K} \partial_{K}}{2 i \pi} K_{\tilde{e}^{L X}}\left(L E, g^{L E}\right)=\delta_{L Y}-{ }^{K} c_{\max }\left(L E, g^{L E}\right) .
$$

The current ${ }^{K} \tilde{e}^{L X}\left(L E, g^{L E}\right)$ is in fact an equivariant Euler-Green current, and its construction is similar to the construction of non-equivariant Euler-Green currents in [BGS5].

Set

$$
Y=L Y \cap X
$$

Let $\tilde{N}=\frac{T L X_{\mid Y}}{T L Y_{\mid Y}+T X_{\mid Y}}$ be the excess normal bundle.

In [B2, Theorems 3.2 and 3.4], we established a formula relating currents on $L X$, $L Y, X$, and $Y$, which is a sort of generalized height pairing formula for the cycles $X$ and $L Y$. In general, this formula reflects the impossibility of forming the product of the currents ${ }^{K} S_{\omega L}$ and ${ }^{K} \tilde{e}^{L X}\left(L E, g^{L E}\right)$. The contribution of the excess normal 
bundle $\tilde{N}$ to the formula appears in the form of a rather mysterious additive genus ${ }^{K} R$ evaluated on $\tilde{N}$.

Let $\left(M, g^{T M}\right)$ be a compact oriented even dimensional spin manifold. Let $L M$ be the loop space of $M$, i.e. the set of smooth maps $s \in S_{1}=\mathbb{R} / \mathbb{Z} \rightarrow x_{s} \in M$. The metric $g^{T M}$ lifts naturally to a metric $g^{T L M}$ on $T L M$. Let $K$ be the Killing vector field which generates the natural action of $S_{1}$ on $L M$, by change of the origin. Then $M$ embeds into $L M$ as the zero set of $K$. In [A], Atiyah and Witten made the fundamental observation that the McKean-Singer formula for the index of the Dirac operator acting on spinors could be written formally as the integral over $L M$ of a $K$-equivariantly closed form on $L M$. By applying formally the localization formulas of Bott [Bo], Berline-Vergne $[\mathrm{BeV}]$ in this infinite dimensional situation, they obtained the right index formula of Atiyah-Singer [AS]. This observation was extended in [B4] to the case of twisted spin complexes. In [B6], a new proof of the localization formulas of [Bo, $\mathrm{BeV}]$ was given, which reproduced in a finite dimensional context the "fantastic cancellations" in local index theory. The geometric origin of such cancellations, and their relation to equivariant cohomology was thus established, at least formally. These considerations have been at the origin of subsequent developments, which include the local families index theorem of [B5, B6].

Let now $\left(X, \omega^{X}\right)$ be a compact complex Kähler manifold, and let $\left(\xi, h^{\xi}\right)$ be a holomorphic Hermitian vector bundle on $X$. Let $\lambda(\xi)=(\operatorname{det} H(X, \xi))^{-1}$ be the inverse of the determinant of the cohomology of $\xi$, and let \|\|$_{\lambda(\xi)}$ be the Quillen metric [Q2, BGS3] on the line $\lambda(\xi)$. The metric \|\|$_{\lambda(\xi)}$ is the product of the $L_{2}$ metric on $\lambda(\xi)$ (which is obtained by identifying $H(X, \xi)$ to the corresponding harmonic forms on $X$ ) by the Ray-Singer analytic torsion of the Dolbeault complex for $\xi$ [RSi].

Let $L X$ be the loop space of $X$, let $K$ be the vector field generating the natural action of $S_{1}$ on $L X$. The Kähler form $\omega^{X}$ lifts to the Kähler form $\omega^{L X}$ of $L X$, and $K$ is now a holomorphic Killing vector field. It was observed in [B8] that the logarithm of the Ray-Singer analytic torsion of $\xi$ could be expressed formally as a normalized integral over $L X$ of the current ${ }^{K} S_{\omega} L X$ paired to the lift to $L X$ of the Chern character form for $\xi \otimes(\operatorname{det} T X)^{1 / 2}$, which was constructed in [B4]. As explained in [B8], this formal analogy was crucial in understanding the generalized anomaly formulas for Quillen metric [BGS3, Sect. 1h]. One of the merits of this approach is that it expresses the logarithm of the Ray-Singer analytic torsion - which is a global invariant of the manifold $X$ - as the formal integral of a local geometrically meaningful current over $L X$.

Let now $k: Y \rightarrow X$ be an embedding of complex manifolds, let $\eta$ be a holomorphic vector bundle on $Y$, and let $(\xi, v)$ be a holomorphic complex of vector bundles over $X$, which provides a resolution of the sheaf $k_{*} \mathscr{Q}_{Y}(\eta)$. By a construction of Grothendieck-Knudsen-Mumford [KMu], we know that the line $\lambda^{-1}(\eta) \otimes \lambda(\xi)$ has a nonzero canonical section $\sigma$. When Kähler metrics are introduced on $T X, T Y, \xi, \eta$, Bismut and Lebeau $[\mathrm{BL} 1,2]$ calculated the Quillen norm $\|\sigma\|_{\lambda^{-1}(\eta) \otimes \lambda(\xi)}$, in terms of Bott-Chern currents on $X, Y$ in the sense of Bismut-Gillet-Soulé [BGS4] and also of the additive genus $R$ of Gillet-Soulé [GS3] associated to the power series

$$
R(x)=\sum_{\substack{n \geq 1 \\ n \text { odd }}}\left(\frac{2 \zeta^{\prime}(-n)}{\zeta(-n)}+\sum_{1}^{n} \frac{1}{j}\right) \zeta(-n) \frac{x^{n}}{n !},
$$

where $\zeta(s)$ is the Riemann zeta function. The additive genus $R$ appears in Gillet-Soule [GS3] in their calculation of the analytic torsion of $\mathbb{P}^{n}(\mathbb{C})$. The genus $R$ reappears in 
a completely different way in Bismut [B7], in a calculation of the generalized analytic torsion forms of a short exact sequence of vector bundles. By putting together the results of [BGS1-5, GS1-3, BL1,2], Gillet and Soule have then established their conjectured Riemann-Roch-Grothendieck formula in Arakelov theory [GS4, 5].

Let $\left(E, g^{E}\right)$ be a holomorphic Hermitian vector bundle on $X$, let $r$ be a holomorphic section on $X$ which exactly vanishes on $Y$, such that $d r_{\mid Y}: N_{Y / X} \rightarrow E_{\mid Y}$ identifies $N_{Y / X}$ and $E_{\mid Y}$. Let $\eta$ be a holomorphic vector bundle on $X$. Then the Koszul complex $\left(\Lambda E^{*} \otimes \eta, i_{r}\right)$ provides a resolution of the sheaf $i_{*} \mathscr{Q}_{Y}\left(\eta_{\mid Y}\right)$.

The purpose of this paper is to show that formally, the formula of Bismut-Lebeau $[B L 1,2]$ which calculates $\log \left(\|\sigma\|_{\lambda^{-1}\left(\eta_{\mid Y}\right) \otimes \lambda\left(\Lambda E^{*} \otimes \eta\right)}^{2}\right)$, can be viewed as a consequence of the intersection formula established in our previous paper [B2]. In particular the genus $R$ of Gillet and Soule is an infinite dimensional version of the genus ${ }^{K} R$ of [B2] described before.

The application of the result of [BL2] to the problem considered in [BL1,2] gives the exact answer. Maddeningly enough, also the intermediary steps in the proofs of Bismut [B2] and Bismut-Lebeau [BL2] are strictly similar. Of course there are many more technical difficulties in [BL2], which are handled by operator theoretic techniques. Nevertheless the comparison with [B2] gives a geometric interpretation to the difficulties in the proof of [BL2]. Namely $L Y$ and $X$ are not transversal in $L X$; there is an excess normal bundle $\tilde{N}$, which is exactly the bundle of loops in the fibres of $N_{Y / X}$ whose integral vanishes. The impossibility of forming the product of currents over $L X$ whose wave front sets are included in $N_{L Y / L X, \mathbb{R}}^{*}$ and $N_{X / L X, \mathbb{R}}^{*}$ is ultimately responsible for the appearance of the genus $R$. Equivalently, one could say that the main result of [BL2] is in part the solution of a hard problem of microlocal analysis in infinite dimensions, and this in the worst possible conditions, i.e. in the case where the excess normal bundle is of infinite dimension.

In retrospect, a whole class of rigorous results concerning finite dimensional BottChern currents [B3, BGS4, 5] and Quillen metrics [BGS1,3, BL1,2] may appear as the shadow of infinitely more complex and richer properties of Bott-Chern currents over loop spaces, whose only disadvantage is that they are ill-defined. Similarly, the remarkable compatibility properties verified by Bott-Chern currents [BGS5] become tautological once their formal relation to currents on loop spaces is understood. The prototype of such a compatibility results is in fact the result of Bismut-Lebeau [BL1,2] itself.

To moderate the somewhat romantic view expressed before, let us also say that all these analogies remain formal, and that they only provide us with a guide to, say, the proof of the main result of [BL2] and not with the proof itself. Certain key objects which appear in the construction of Quillen metrics are invisible in the functional integration formalism, and still play a key role in [BL2]: among these invisible objects, the cohomology groups themselves... . This is why, in this paper, we have been very careful in distinguishing "formal" results from rigorous results, although even formal results may have proofs.

This paper is organized as follows. In Sect. 1, we introduce the loop space formalism, and we show how certain well-defined Bott-Chern currents on a complex manifold $X$ may be viewed as generalized restrictions to $X$ of $K$-equivariant BottChern currents on $L X$. In Sect.2, we briefly recall the construction of the Quillen metric on the determinant of the cohomology [Q2, BGS3], and we formulate the problem considered in Bismut-Lebeau [BL1,2]. Finally, in Sect. 3, we make a short 
parallel between the result and proofs of [BL2] and [B2], which leads us in particular to a formal proof of the main result of $[\mathrm{BL} 1,2]$.

In the whole paper, we use the formalism of Mathai-Quillen [MQ], and also the results and notation of the companion paper [B2].

\section{Bott-Chern Currents in Finite Dimensions and Euler-Green Currents in Infinite Dimensions}

The purpose of this section is to show that certain well-defined Bott-Chern currents on finite dimensional complex manifolds can be viewed formally as "restrictions" to the manifold of equivariant Euler-Green currents on the corresponding loop spaces. In particular we relate the lack of local integrability of these well-defined Bott-Chern currents to the presence of an infinite dimensional excess normal bundle. The results of this section are only mildly formal.

The Bott-Chern currents have been rigorously constructed on finite dimensional manifolds by Bismut-Gillet-Soulé [BGS4]. Euler-Green currents have been constructed in [BGS5] on finite dimensional manifolds, and equivariant Euler-Green currents on finite dimensional manifolds were constructed in [B2].

This section is organized as follows. In a), we introduce the loop space formalism. In b), we express certain well-defined currents over a finite dimensional complex manifold in terms of ill-defined currents on the corresponding loop space.

a) Complex Embeddings and Loop Spaces. Let $X$ be a compact complex manifold. Let $\mathbf{J}^{T X}$ be the complex structure on the real tangent bundle $T_{\mathbb{R}} X$.

Let $L X$ be the set of smooth maps $t \in S_{1}=\mathbb{R} / \mathbb{Z} \rightarrow x_{t} \in X . L X$ will be called the loop space of $X$.

If $x \in L X$, we identify the real tangent space $\left(T_{\mathbb{R}} L X\right)_{x}$ with the vector space of smooth sections $t \in S_{1} \rightarrow U_{t} \in\left(T_{\mathbb{R}} X\right)_{x_{t}}$. The complex structure $\mathbf{J}^{T X}$ induces the obvious complex structure $\mathbf{J}^{T L X}: U \in\left(T_{\mathbb{R}} L X\right) \rightarrow \mathbf{J}^{T X} U \in T_{\mathbb{R}} L X$. One easily verifies that the complex structure $\mathbf{J}^{T L X}$ is integrable. Then $L X$ can be considered as a complex manifold. If $x \in L X$, the complex tangent space $T_{x} L X$ can be identified with the vector space of smooth maps $t \in S_{1} \rightarrow U_{t} \in T_{x_{t}} X$.

If $x \in X$, we identify $x$ with the constant loop $t \in S_{1} \rightarrow x$. Then $X$ is a complex submanifold of $L X$. Let $f$ be the embedding $X \rightarrow L X$.

For $s \in S_{1}, x \in L X$, set $\left(k_{s} x\right)_{t}=x_{s+t}$. Then $\left(k_{s}\right)_{s \in S_{1}}$ is a group of diffeomorphisms of $L X$. Its generating vector field $K$ is given by

$$
K(x)_{t}=\frac{d x_{t}}{d t}
$$

Clearly, $K$ is a holomorphic vector field on $L X$. Also

$$
X=\{x \in L X ; K(x)=0\} .
$$

Let $g^{T X}$ be a Hermitian metric on $T X$ such that $\left(X, g^{T X}\right)$ is a Kähler manifold. Let $\langle,\rangle_{T X}$ be the corresponding Hermitian product on $T X$. Let $\omega^{X}$ be the Kähler form

$$
U, V \in T_{\mathbb{R}} X \rightarrow \omega^{X}(U, V)=\left\langle U, \mathbf{J}^{T X} V\right\rangle_{T X} .
$$

If $x \in L X$, if $U, V \in\left(T_{\mathbb{R}} L X\right)_{x}$, set

$$
\langle U, V\rangle_{T L X}=\int_{0}^{1}\left\langle U_{t}, V_{t}\right\rangle_{T_{x_{t}} X} d t .
$$


Then (1.4) defines a Hermitian product $g^{T L X}$ on $T L X$. The manifold $\left(L X, g^{T L X}\right)$ is still Kähler. The corresponding Kähler form $\omega^{L X}$ is given by

$$
U, V \in T_{\mathbb{R}} L X \rightarrow \omega^{L X}(U, V)=\int_{0}^{1} \omega_{x_{t}}^{X}\left(U_{t}, V_{t}\right) d t
$$

Of course the metric $g^{T L X}$ induces the metric $g^{T X}$ on $T X$, and $\omega^{X}$ is the restriction of $\omega^{L X}$ to $X$. Moreover the vector field $K$ is a Killing vector field with respect to the metric $g^{T L X}$.

Let $E$ be a holomorphic vector bundle on $X$. We assume that $\operatorname{dim} E \leq \operatorname{dim} X$. Let $r$ be a holomorphic section of $E$ over $X$. Set

$$
Y=\{x \in X ; r(x)=0\} .
$$

We assume that for any $y \in Y$, the linear map $d r(y): T_{y} X \rightarrow E_{y}$ has maximal rank $\operatorname{dim}(E)$. Thus $Y$ is a complex submanifold of $X$.

Let $k$ be the embedding $Y \rightarrow X$. Let $N_{Y / X}$ be the normal bundle to $Y$ in $X$. Then $d r: N_{Y / X} \rightarrow E_{\mid Y}$ is an identification of holomorphic vector bundles over $Y$.

The vector bundle $T Y$ inherits the metric $g^{T Y}$ from the metric $g^{T X}$. The corresponding Kähler form $\omega^{Y}$ on $Y$ is the restriction to $Y$ of $\omega^{X}$. The manifold $\left(Y, g^{T Y}\right)$ is also a Kähler manifold.

By identifying $N_{Y / X}$ with the orthogonal bundle to $T Y$ in $T X_{\mid Y}, N_{Y / X}$ inherits a metric $g^{N_{Y / X}}$.

Let $g^{E}$ be a Hermitian metric on $E$. We make the assumption that the identification $d r_{\mid Y}: N_{Y / X} \rightarrow E$ also identifies the metrics. One verifies easily that there exists a metric $g^{E}$ on $E$ such that this assumption is verified.

Let $s$ be the section of $E_{\mathbb{R}}, s=r+\bar{r}$. Let $\nabla^{E}$ be the holomorphic Hermitian connection on $\left(E, g^{E}\right)$. Let $L E$ be the set of smooth maps $t \in S_{1}=\mathbb{R} / \mathbb{Z} \rightarrow e_{t} \in E$. Then $L E$ is a vector bundle over $L X$. If $x \in L X$, the fibre $(L E)_{x}$ is the set of $e \in L E$ such that if $s \in S_{1}, e_{t} \in E_{x_{t}}$. By proceeding as before, we see that $L E$ can be considered formally as a holomorphic vector bundle on $L X$. If $e, e^{\prime} \in(L E)_{x}$, set

$$
\left\langle e, e^{\prime}\right\rangle_{g^{L E}}=\int_{0}^{1}\left\langle e_{t}, e_{t}^{\prime}\right\rangle_{g_{x_{t}}^{E}} d t
$$

Then $g^{L E}$ is a Hermitian metric on $L E$. Let $\nabla^{L E}$ be the holomorphic Hermitian convention on $\left(L E, g^{L E}\right)$. One verifies easily that if $x \in L X, e \in L E$, if $U \in$ $\left(T_{\mathbb{R}} L X\right)_{x}$, then

$$
\left(\nabla_{U}^{L E} e\right)_{t}=\nabla_{U_{t}}^{E} e_{t}
$$

If $s \in S_{1}, e \in L E$, set

$$
\left(k_{s}^{L E} e\right)_{t}=e_{s+t} .
$$

Then $\left(k^{L E}\right)_{s \in S_{1}}$ is a group of holomorphic unitary isomorphisms of $L E$, which lifts the group $\left(k_{s}\right)_{s \in S_{1}}$ to $L E$. Let $K^{L E}$ be the vector field on $L E$ which generates the group $\left(k_{s}^{L E}\right)_{s \in S_{1}}$. The vector field $K^{L E}$ lifts $K$ to $L E$.

We now use the notation of [B2, Sect. 2c)]. Namely let $J^{L E}$ be the horizontal part of $K^{L E}$ with respect to the connection $\nabla^{L E}$. The tensor $J^{L E}$ is then a skew-adjoint section of End $(L E)$. 
Take $x \in L X, e \in(L E)_{x}$. Let $\frac{D^{E}}{D t}$ be the covariant differentiation operator along the loop $t \rightarrow x_{t}$ with respect to the connection $\nabla^{E}$. One verifies easily that

$$
\left(J^{L E} e\right)_{t}=\frac{D^{L E} e}{D t} .
$$

The section $r$ of $E$ lifts to a section of $L E$ over $L X$. In fact if $x \in L X$, for any $t$, $r_{x_{t}} \in L E_{x_{t}}$. We still denote by $r$ this section of $L E$ over $L X$. Similarly the section $s=r+\bar{r}$ of $E_{\mathbb{R}}$ over $X$ extends to a section of $L E_{\mathbb{R}}$ over $L X$. Of course $r, s$ are $K^{L E}$-invariant sections of $L E, L E_{\mathbb{R}}$.

Let $L Y$ be the loop space of $Y$. Clearly

$$
L Y=\left\{x \in L X ; r_{x}=0\right\} .
$$

Also

$$
Y=L Y \cap X
$$

If $y \in L Y$, the normal bundle $\left(N_{L Y / L X}\right)_{y}$ can be identified with $L N_{Y / X}$, i.e. with the set of smooth maps $t \in S_{1} \rightarrow n_{t} \in N_{Y / X}$, with $n_{t} \in\left(N_{Y / X}\right)_{y_{t}}$. If $y \in L Y$, the map $d r_{y}: N_{L Y / L X, y} \rightarrow(L E)_{L Y, y}$ is an identification of holomorphic vector bundles over $L Y$.

Similarly if $y \in Y$, let $\tilde{N}_{y}$ be the set of smooth maps $t \in S_{1} \rightarrow \tilde{n}_{t} \in N_{Y / X, y}$ such that $\int_{0}^{1} \tilde{n}_{t} d t=0$. Then we have an identification of holomorphic vector bundles
over $Y$

$$
N_{L Y / L X_{\mid Y}}=N_{Y / X} \oplus \tilde{N}
$$

b) Accumulating Evidence: Some Finite Dimensional Formulas.

1) Evaluation of ${ }^{K} c_{\max }^{-1}\left(N_{X / L X}, g^{N_{X / L X}}\right)$. Take $x \in X$. Then one easily verifies that $\left(N_{X / L X}\right)_{x}$ can be identified with the set of smooth maps $t \in S_{1} \rightarrow U_{t} \in T_{x} X$ such that $\int_{0}^{1} U_{t} d t=0$. Also $N_{X / L X}$ inherits the obviously $L_{2}$ metric $g^{N_{X / L X}}$ constructed in (1.7). If $U \in N_{X / L X}$, then

$$
J^{N_{X / L X}} U=\frac{d U}{d t}
$$

The eigenvalues of $J^{N_{X / L X}}$ are obviously given by $2 i k \pi\left(k \in Z^{*}\right)$. Therefore at least formally, $N_{X / L X}$ splits holomorphically as a countable direct sum indexed by $k \in \mathbb{Z}^{*}$,

$$
N_{X / L X}=\bigoplus_{k \in \mathbb{Z}^{*}} T X
$$

Also the splitting (1.15) identifies the metrics. On the $k^{\text {th }}$ copy of $T X, J^{N_{X / L X}}$ acts by multiplication by $2 i k \pi$.

Let $\nabla^{T X}$ be the holomorphic Hermitian connection on $\left(T X, g^{T X}\right)$, and let $R^{T X}$ be its curvature. Similarly let $\nabla^{N_{X / L X}}$ be the holomorphic Hermitian connection on $\left(N_{X / L X}, g^{N_{X / L X}}\right)$ and let $R^{N_{X / L X}}$ be its curvature.

Set

$$
{ }^{K} R^{N_{X / L X}}=J^{N_{X / L X}}+R^{N_{X / L X}} .
$$


By [B2, Eq. (1.16)], we have the identity

$$
{ }^{K} c_{\max }^{-1}\left(N_{X / L X}, g^{N_{X / L X}}\right)=\frac{1}{\operatorname{det}\left(-\frac{{ }^{K} R^{N_{X / L X}}}{2 i \pi}\right)} .
$$

Using (1.15), we get the formal formula

$$
{ }^{K} c_{\max }^{-1}\left(N_{X / L X}, g^{N_{X / L X}}\right)=\prod_{k \in \mathbb{Z}^{*}} \frac{1}{\operatorname{det}\left(-\frac{2 i k \pi+R^{T X}}{2 i \pi}\right)} .
$$

Equivalently

$$
{ }^{K} c_{\max }^{-1}\left(N_{X / L X}, g^{N_{X / L X}}\right)=\frac{1}{\left(\prod_{1}^{+\infty}-k^{2}\right)^{\operatorname{dim} X}} \frac{1}{\prod_{1}^{+\infty} \operatorname{det}\left(1+\frac{\left(R^{T X}\right)^{2}}{4 \pi^{2} k^{2}}\right)}
$$

Let $\hat{A}$ be the complex Hirzebruch polynomial. Namely if $B$ is a $(q, q)$ matrix, set

$$
\hat{A}(B)=\operatorname{det}\left[\frac{B / 2}{\sinh (B / 2)}\right] \text {. }
$$

Recall that

$$
\frac{\sinh (x / 2)}{x / 2}=\prod_{1}^{+\infty} \operatorname{det}\left(1+\frac{x^{2}}{4 k^{2} \pi^{2}}\right)
$$

Let $C$ be the infinite constant

$$
C=\prod_{1}^{+\infty}\left(-k^{2}\right)
$$

From (1.17)-(1.21), we deduce the following result.

Proposition 1.1. One has the formal identity of forms on $X$

$$
{ }^{K} c_{\max }^{-1}\left(N_{X / L X}, g^{N_{X / L X}}\right)=C^{-\operatorname{dim} X} \hat{A}\left(R^{T X}\right) .
$$

The identity (1.22) is used in a crucial way by Atiyah and Witten [A] to show that formally, one can derive the index theorem for the Dirac operator acting on spinors from a localization formula from $L X$ to $X$ in equivariant cohomology.

2. Evaluation of ${ }^{K} c_{\max }\left(L E_{\mid X}, g^{L E_{\mid X}}\right)$ on $X$. Let $R^{E}$ be the curvature of $\nabla^{E}$ and let $R^{L E}$ be the curvature of $\nabla^{L E}$. Then if ${ }^{K} R^{L E}=J^{L E}+R^{L E}$, we have the identity of forms on $L X$

$$
{ }^{K} c_{\max }\left(L E, g^{L E}\right)=\operatorname{det}\left(-\frac{{ }^{K} R^{L E}}{2 i \pi}\right) .
$$

The eigenvalues of $J^{L E}$ acting on $L E_{\mid X}$ are given by $2 i k \pi(k \in \mathbb{Z})$. By proceeding as in (1.17)-(1.21), we get the identity of forms on $X$

$$
f^{* K} c_{\max }\left(L E, g^{L E}\right)=\left(\frac{i C}{2 \pi}\right)^{\operatorname{dim} E} \operatorname{det}\left(e^{R^{E} / 2}-e^{-R^{E} / 2}\right) .
$$


Let $\operatorname{ch}\left(E, g^{E}\right)=\operatorname{Tr}\left[\exp \left(\frac{-R^{E}}{2 i \pi}\right)\right]$ be the Chern character form on $X$ associated to $\left(E, g^{E}\right)$. Let $\Lambda E^{*}$ be the exterior algebra of $E^{*}$. The metric $g^{E}$ induces a metric $g^{\Lambda E^{*}}$ on $\Lambda E^{*}$. Set

$$
\operatorname{ch}\left(\Lambda E^{*}, g^{\Lambda E^{*}}\right)=\sum_{0}^{\operatorname{dim} E}(-1)^{p} \operatorname{ch}\left(\Lambda^{p} E^{*}, g^{\Lambda^{p} E^{*}}\right) .
$$

Let $\varphi$ be the algebra homomorphism from $\Lambda^{\text {even }} T_{\mathbb{R}}^{*} X$ into itself which to $\omega \in$ $\Lambda^{2 p}\left(T_{\mathbb{R}}^{*} X\right)$ associates $(2 i \pi)^{-p} \omega \in \Lambda^{2 p}\left(T_{\mathbb{R}}^{*} X\right)$.

Then one has the easy formula

$$
\operatorname{ch}\left(\Lambda E^{*}, g^{\Lambda E^{*}}\right)=\varphi \operatorname{det}\left(1-e^{R^{E}}\right)
$$

From (1.24), (1.26), we deduce the following result.

Proposition 1.2. One has the formal identity of forms on $X$

$$
{ }^{K} c_{\max }\left(L E_{\mid X}, g^{L E_{\mid X}}\right)=\left(\frac{C}{2 \pi i}\right)^{\operatorname{dim} E}\left[\varphi^{-1} \operatorname{ch}\left(\Lambda E^{*}, g^{\Lambda E^{*}}\right)\right] e^{-\frac{\operatorname{Tr}\left[R^{E}\right]}{2}} .
$$

3. Evaluation of $f^{*} s^{*}{ }^{K} c_{T}\left(L E, g^{L E}\right)$ on $X$. By proceeding as in (1.22)-(1.24), we get the identity of forms on $X$

$$
\begin{aligned}
f^{*} s^{* K} c_{T}\left(L E, g^{L E}\right)= & \left(\frac{i C}{2 \pi}\right)^{\operatorname{dim} E} \frac{\partial}{\partial b}\left[\operatorname{det}\left(e^{\frac{R^{E}+2 \pi i b}{2}}-e^{-\frac{R^{E}+2 \pi i b}{2}}\right)\right. \\
& \left.s^{*} \exp \left\{-T\left(\frac{\left|Y^{2}\right|}{2}+\left(R^{E}+2 \pi b \mathbf{J}^{E}\right)^{-1}\right)\right\}\right]_{b=0} .
\end{aligned}
$$

The exterior algebra $\Lambda E^{*}$ is a $c\left(E_{\mathbb{R}}\right)$-Clifford module. If $e \in E_{\mathbb{R}}$, let $\hat{c}(e)$ denote the corresponding Clifford multiplication operator. If $e^{\prime} \in \bar{E}$, let $e^{\prime *}$ be the element in $E^{*}$ corresponding to $e^{\prime}$ by the metric $g^{E}$. Then if $e \in E, e^{\prime} \in \bar{E}$, we have the identities

$$
\hat{c}(e)=\sqrt{2} i_{e} ; \quad \hat{c}\left(e^{\prime}\right)=-\sqrt{2} e^{* *} \wedge .
$$

Let $A_{T}$ be the superconnection on $\Lambda E^{*}$ in the sense of Quillen [Q1],

$$
A_{T}=\nabla^{\Lambda E^{*}}+\sqrt{T} \sqrt{-1} \frac{\hat{c}(s)}{\sqrt{2}} .
$$

Let $N_{H}$ be the number operator of $\Lambda E^{*}$, i.e. $N_{H}$ acts on $\Lambda^{p}\left(E^{*}\right)$ by multiplication by $p$. By [B3, Eqs. (3.142), (3.143), (4.21) and (4.22)], we know that

$$
\begin{aligned}
\operatorname{Tr}_{s}\left[N_{H} \exp \left(-A_{T}^{2}\right)\right]= & \frac{\partial}{\partial b}\left[\operatorname{det}\left(1-\exp \left(R^{E}+b\right)\right)\right. \\
& \left.s^{*} \exp \left\{-T\left(\frac{|Y|^{2}}{2}+\left(R^{E}-b i \mathbf{J}^{E}\right)^{-1}\right)\right\}\right]_{b=0} .
\end{aligned}
$$

Using (1.28)-(1.30), we get the following result. 
Proposition 1.3. The following formal equality of forms on $X$ holds

$$
\begin{aligned}
& f^{*} s^{* K} c_{T}\left(L E, g^{L E}\right) \\
& \quad=\left(\frac{C}{2 \pi i}\right)^{\operatorname{dim} E} 2 \pi i \operatorname{Tr}_{s}\left[\left(N_{H}-\frac{\operatorname{dim} E}{2}\right) \exp \left(-A_{T}^{2}\right)\right] e^{-1 / 2 \operatorname{Tr}\left[R^{E}\right]} .
\end{aligned}
$$

4. Evaluation of ${ }^{K} \tilde{e}^{X}\left(L E, g^{L E}\right)$. Let $k$ be the embedding $Y \rightarrow X$. The complex

$$
\left(\Lambda E^{*}, i_{r}\right): 0 \rightarrow \Lambda^{\operatorname{dim} E} E^{*} \underset{i_{r}}{\rightarrow} \ldots \underset{i_{r}}{\rightarrow} \Lambda^{0} E^{*}=\mathbb{C} \rightarrow 0
$$

provides a resolution of the sheaf $k_{*} \mathscr{Q}_{Y}$. Moreover $\Lambda E^{*}=\bigoplus_{0}^{\operatorname{dim} E} \Lambda^{p} E^{*}$ is naturally equipped with a Hermitian metric $g^{\Lambda E^{*}}$. Therefore, by [BGS4], we can construct the associated current $T^{X}\left(\Lambda E^{*}, g^{\Lambda E^{*}}\right)$ on $X$. This current was also described in [B1, Sect. 1i)].

If $B$ is a $(q, q)$ matrix, set

$$
\operatorname{Td}(B)=\operatorname{det}\left(\frac{B}{1-e^{-B}}\right), \quad\left(\hat{A}^{-1}\right)^{\prime}(B)=\frac{\partial}{\partial b}\left[\hat{A}^{-1}(B+b)\right]_{b=0} .
$$

Recall that $c_{1}\left(N_{Y / X}, g^{N_{Y / X}}\right)=-\frac{\operatorname{Tr}\left[R^{N_{Y / X}}\right]}{2 i \pi}$.

In view of [B3, Theorem 3.2], or of [B2, Theorem 2.18] and (1.30), we are entitled to set the following definition.

Definition 1.4. For $s \in \mathbb{C}, 0<\operatorname{Re}(s)<\frac{1}{2}$, let $\tilde{R}^{X}\left(\Lambda E^{*}, g^{\Lambda E^{*}}\right)$ be the current on $X$

$$
\begin{aligned}
\tilde{R}^{X}\left(\Lambda E^{*}, g^{\Lambda E^{*}}\right)(s)= & \frac{1}{\Gamma(s)} \int_{0}^{+\infty} u^{s-1}\left(\varphi\left[\operatorname{Tr}_{s}\left(N_{H}-\frac{\operatorname{dim} E}{2}\right) \exp \left(-A_{u}^{2}\right)\right]\right. \\
& \left.+\left(\hat{A}^{-1}\right)^{\prime}\left(N_{Y / X}, g^{N_{Y / X}}\right) e^{-c_{1} / 2\left(N_{Y / X}, g^{N_{Y / X}}\right)} \bar{\partial}_{Y}\right) d u
\end{aligned}
$$

Then $\tilde{R}^{X}\left(\Lambda E^{*}, g^{\Lambda E^{*}}\right)(s)$ extends to a holomorphic function of $s \in \mathbb{C}$ near $s=0$.

Definition 1.5. Set

$$
\tilde{T}^{X}\left(\Lambda E^{*}, g^{\Lambda E^{*}}\right)=\frac{\partial}{\partial s} \tilde{R}^{X}\left(\Lambda E^{*}, g^{\Lambda E^{*}}\right)(0)
$$

Let $P_{Y}^{X}$ be the set of sums of currents on $X$ of type $(p, p)$ whose wave front set in included in $N_{Y / X, \mathbb{R}}^{*}$, and let $P_{Y}^{X, 0}$ be the set of currents $\alpha \in P_{Y}^{X}$ such that there exist currents $\beta, \gamma$ on $X$ whose wave front set is included in $N_{Y / X, \mathbb{R}}^{*}$, for which $\alpha=\partial \beta+\bar{\partial} \gamma$.

We identify $X$ to the zero section of $E$. Recall that the current $\tilde{e}\left(E, g^{E}\right)$, which lies in $P_{X}^{E}$, was constructed in [BGS5, Sect. 3f). It is of complex type (dim $E-1, \operatorname{dim} E-$ 1). The pulled-back current $s^{*} \tilde{e}\left(E, g^{E}\right)$ on $X$ is well defined, and lies in $P_{Y}^{X}$. 
Theorem 1.6. The current $\tilde{T}^{X}\left(\Lambda E^{*}, g^{\Lambda E^{*}}\right)$ lies in $P_{Y}^{X}$. Moreover the following identity of current holds

$$
\tilde{T}^{X}\left(\Lambda E^{*}, g^{\Lambda E^{*}}\right)=T^{X}\left(\Lambda E^{*}, g^{\Lambda E^{*}}\right) \quad \text { in } \quad P_{Y}^{X} / P_{Y}^{X, 0}
$$

Also,

$$
\tilde{T}^{X}\left(\Lambda E^{*}, g^{\Lambda E^{*}}\right)=\operatorname{Td}^{-1}\left(E, g^{E}\right) s^{*} \tilde{e}\left(E, g^{E}\right) \quad \text { in } \quad P_{Y}^{X} P_{Y}^{X, 0} .
$$

Proof. To construct the current $T^{X}\left(\Lambda E^{*}, g^{\Lambda E^{*}}\right)$, one should essentially replace in (1.33) $N_{H}-\frac{\operatorname{dim} E}{2}$ by $N_{H}$. By using [B3, Theorems 2.4 and 3.2], (1.35) follows. The identity (1.36) is a consequence of (1.35) and of [BGS5, Theorem 3.17].

Remark 1.7. By [BGS4, Sect. 3], the current $T^{X}\left(\Lambda E^{*}, g^{\Lambda E^{*}}\right)$ is in general not locally integrable near $Y$. The singularity of $T^{X}\left(\Lambda E^{*}, g^{\Lambda E^{*}}\right)$ is described in [BGS4, Theorems 3.3 and 3.4].

Recall that when $X$ and $L X$ are finite dimensional, the current ${ }^{K} \tilde{e}^{X}\left(L E, g^{L E}\right)$ was constructed in [B2, Sects. 2e) and 2f)].

Using [B2, Definition 2.19] and from Proposition 1.3, we get the following formal result.

Theorem 1.8. The following formal identity of currents on $X$ holds

$$
{ }^{K} \tilde{e}^{X}\left(L E, g^{L E}\right)=\left(\frac{C}{2 \pi i}\right)^{\operatorname{dim} E} 2 \pi i \varphi^{-1}\left(\tilde{T}^{X}\left(\Lambda E^{*}, g^{\Lambda E^{*}}\right)\right) e^{-1 / 2 \operatorname{Tr}\left[R^{E}\right]} .
$$

Identity (1.37) is of fundamental interest. In fact, by a direct study similar to what is done in [BGS4, Sect. 3] for the current $T^{X}\left(\Lambda E^{*}, g^{\Lambda E^{*}}\right)$, we see that in general the current $\tilde{T}^{X}\left(\Lambda E^{*}, g^{\Lambda E^{*}}\right)$ is not locally integrable. More precisely, we find that near $Y$, if $Z$ is a coordinate normal to $Y$ in $X, \tilde{T}^{X}\left(\Lambda E^{*}, g^{\Lambda E^{*}}\right)$ behaves like

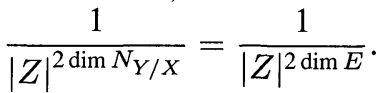

On the other hand, if we apply formally the results of [B2, Sects. 2e) and 2f)] to the current ${ }^{K} \tilde{e}^{X}\left(L E, g^{L E}\right)$, we find that ${ }^{K} \tilde{e}^{X}\left(L E, g^{L E}\right)$ is not locally integrable on $X$, and, more precisely, that the current ${ }^{K} \tilde{e}^{X}\left(L E, g^{L E}\right)$ also behaves near $Y$ like $\frac{1}{|Z|^{2 \operatorname{dim} E}}=\frac{1}{|Z|^{2 \operatorname{dim} N_{Y / X}}}$. That the formal singularity of the current ${ }^{K} \tilde{e}^{X}\left(L E, g^{L E}\right)$ near $Y$ coincides with the singularity of the current in the right-hand side of (1.37) near $Y$ is now a tautological consequence of (1.37).

In [B2, Remark 2.23], if $L X$ was instead a finite dimensional manifold, the fact that the current ${ }^{K} \tilde{e}^{X}\left(L E, g^{L E}\right)$ is not locally integrable near $Y$ is essentially related to the non-transversality of $L Y$ and $X$ in $L X$, and more precisely to the presence of a nonzero excess normal bundle $\tilde{N}$. At least formally, we have given a simple reformulation of a result on the well-defined current $\tilde{T}^{X}\left(\Lambda E^{*}, g^{\Lambda E^{*}}\right)$ on $X$ in terms of the geometry of the infinite dimensional manifold $L X$.

\section{Quillen Metrics and Resolutions}

In this short section, we briefly construct the Quillen metrics on the determinant of the cohomology, and we also describe the problem which is solved in Bismut-Lebeau 
[BL1,2]. We make the same assumptions as in Sect. 1. We also use the notation of Sect. 1.

a) The Quillen Metric on the Inverse of the Determinant of the Cohomology. To simplify the arguments which follow, we will assume that $T_{\mathbb{R}} X$ and $E_{\mathbb{R}}$ are spin vector bundles. By $[\mathrm{H}]$, we know that it is equivalent to assume that the line bundles $\operatorname{det} T X$ and $\operatorname{det} E$ have square roots $(\operatorname{det} T X)^{1 / 2}$ and $(\operatorname{det} E)^{1 / 2}$. Observe that on $Y$,

$$
\operatorname{det} T Y=(\operatorname{det} T X)_{\mid Y} \otimes(\operatorname{det} E)_{Y}^{-1} \text {. }
$$

Therefore $T_{\mathbb{R}} Y$ is also a spin vector bundle.

Let $\xi$ be a holomorphic vector bundle on $X$. Let $h^{\xi}$ be a Hermitian metric on $\xi$.

Let $\lambda(\xi)$ be the complex line

$$
\lambda(\xi)=\bigotimes_{i=0}^{\operatorname{dim} X}\left(\operatorname{det} H^{i}(X, \xi)\right)^{(-1)^{i+1}}
$$

Let $\mathbb{H}$ be the vector space of smooth sections of $\Lambda\left(T^{*(0,1)} X\right) \otimes \xi$ over $X$. For $0 \leq p \leq \operatorname{dim} X$, let $\mathbb{H}^{p}$ be the vector space of smooth sections of $\Lambda^{p}\left(T^{*(0,1)} X\right) \otimes \xi$. Then

$$
\mathbb{H}=\bigoplus_{p=0}^{\operatorname{dim} X} \mathbb{H}^{p}
$$

Let $N_{V}^{X}$ be the number operator of $\mathbb{H}$. Then $N_{V}^{X}$ acts on $\mathbb{H}^{p}$ by multiplication by $p$. Let $d v_{X}$ be the volume form on $X$ associated to the metric $g^{T X}$. We equip $\mathbb{H}$ with the Hermitian product

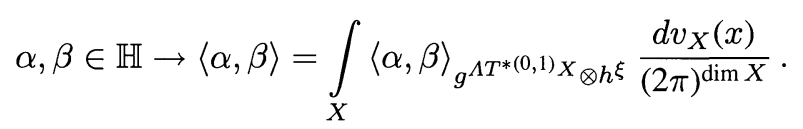

Let $\bar{\partial}^{X}$ be the Dolbeault operator acting on $\mathbb{H}$, and let $\bar{\partial}^{X^{*}}$ be its formal adjoint with respect to the Hermitian product (2.4). The Laplacian $\square^{X}$ is given by

$$
\square^{X}=\left(\bar{\partial}^{X}+\bar{\partial}^{X^{*}}\right)^{2}
$$

The operator $\square^{X}$ preserves the splitting $\mathbb{H}=\bigoplus_{0}^{\operatorname{dim} X} \mathbb{H}^{p}$.

By Hodge theory, we know that we have an identification of $\mathbb{Z}$-graded vector spaces

$$
H(X, \xi) \cong \operatorname{Ker}\left[\square^{X}\right]
$$

As a vector subspace of $\mathbb{H}, \operatorname{Ker}\left(\square^{X}\right)$ inherits a Hermitian product from the Hermitian product (2.4). Using (2.2), (2.6), we may equip the line $\lambda(\xi)$ with the corresponding Hermitian metric, which we denote ||$_{\lambda(\xi)}$.

Let $P$ be the orthogonal projection operator from $\mathbb{H}$ on $\operatorname{Ker}\left(\square^{X}\right)$. Set $P^{\perp}=1-P$.

The vector space $\mathbb{H}$ being $\mathbb{Z}$-graded is also $\mathbb{Z}_{2}$ graded. Let $\operatorname{Tr}_{s}$ be the corresponding supertrace in the sense of Quillen [Q1], which is evaluated on trace class elements of $\operatorname{End}(\mathbb{H})$.

Definition 2.1. For $s \in \mathbb{C}, \operatorname{Re}(s)>\operatorname{dim} X$, set

$$
\theta_{X}^{\xi}(s)=-\operatorname{Tr}_{s}\left[\left(N_{V}^{X}-\frac{\operatorname{dim} X}{2}\right)\left(\square^{X}\right)^{-s} P^{\perp}\right] .
$$


By Seeley [Se], $\theta_{X}^{\xi}(s)$ extends to a function which is holomorphic near $s=0$. Note that classically

$$
\operatorname{Tr}_{s}\left[\left(\square{ }^{X}\right)^{-s} P^{\perp}\right]=0 .
$$
The introduction in (2.7) of the factor $-\frac{\operatorname{dim} X}{2}$ is essentially done for aesthetic
reasons.

Clearly, for $s \in \mathbb{C}, \operatorname{Re}(s)>\operatorname{dim} X$, we have the identity

$$
\theta_{X}^{\xi}(s)=-\frac{1}{\Gamma(s)} \int_{0}^{+\infty} t^{s-1} \operatorname{Tr}_{s}\left[\left(N_{V}^{X}-\frac{\operatorname{dim} X}{2}\right) \exp \left(-t \square^{X}\right) P^{\perp}\right] d t .
$$

Definition 2.2. The Quillen metric \|\|$_{\lambda(\xi)}$ on the line $\lambda(\xi)$ is defined by the formula

$$
\|\|_{\lambda(\xi)}=\|_{\lambda(\xi)} \exp \left\{-\frac{1}{2} \frac{\partial \theta_{X}^{\xi}}{\partial s}(0)\right\} \text {. }
$$

The factor $\exp \left\{-\frac{1}{2} \frac{\partial \theta_{X}^{\xi}}{\partial s}(0)\right\}$ is called the Ray-Singer analytic torsion of the complex $(\mathbb{H}, \bar{\partial})[\mathrm{RSi}]$.

b) Quillen Metrics and Resolutions. Recall that $k$ denotes the embedding $Y \rightarrow X$. Let $\eta$ be a holomorphic Hermitian vector bundle on $X$. Let $a$ be the restriction map $\mathscr{Q}_{X}(\eta) \rightarrow k_{*} \mathscr{G}_{Y}\left(\eta_{\mid Y}\right)$. On $X$ we have the exact sequence of sheaves

$$
\begin{aligned}
0 & \rightarrow \mathscr{\odot}_{X}\left(\Lambda^{\operatorname{dim} E}\left(E^{*}\right) \otimes \eta\right) \underset{i_{r}}{\rightarrow} \mathscr{Q}_{X}\left(\Lambda^{\operatorname{dim} E-1}\left(E^{*}\right) \otimes \eta\right) \\
& \rightarrow \ldots \underset{i_{r}}{\rightarrow} \mathscr{Q}_{X}(\eta) \underset{a}{\rightarrow} k^{*} \mathscr{Q}_{Y}\left(\eta_{\mid Y}\right) \rightarrow 0 .
\end{aligned}
$$

For $p \in \mathbb{N}, 0 \leq p \leq \operatorname{dim} E, \Lambda^{p} E^{*} \otimes \eta$ is a holomorphic vector bundle on $X$. Let $\lambda\left(\Lambda^{p} E^{*} \otimes \eta\right)$ be the corresponding inverse determinant line of the cohomology of $\mathscr{Q}_{X}\left(\Lambda^{p} E^{*} \otimes \eta\right)$, which we construct as before.

Set

$$
\lambda\left(\Lambda E^{*} \otimes \eta\right)=\bigotimes_{p=0}^{\operatorname{dim} E}\left(\lambda\left(\Lambda^{p} E^{*} \otimes \eta\right)\right)^{(-1)^{p}} .
$$

Also $\eta_{\mid Y}$ is a holomorphic vector bundle on $Y$. Let $\lambda\left(\eta_{\mid Y}\right)$ be the associated inverse determinant line of the cohomology of $Q_{Y}(\eta)$.

By a well-known construction of Grothendieck-Knudsen-Mumford [KMu], the lines $\lambda\left(\Lambda E^{*} \otimes \eta\right)$ and $\lambda\left(\eta_{\mid Y}\right)$ are canonically isomorphic. Let $\sigma$ be the canonical nonzero section of $\lambda^{-1}\left(\eta_{\mid Y}\right) \otimes \lambda\left(\Lambda E^{*} \otimes \eta\right)$ which defines this canonical isomorphism. Of course $\sigma$ depends explicitly on the section $r$ of $E$.

Let $g^{\eta}$ be a Hermitian metric on $\eta$. The vector bundles $T X, \Lambda^{p} E^{*} \otimes \eta(0 \leq p \leq$ $\operatorname{dim} E$ ) on $X$, and $T Y, \eta_{\mid Y}$ on $Y$ are now naturally equipped with Hermitian metrics. Let \|\|$_{\lambda\left(\Lambda^{p} E^{*} \otimes \eta\right)},\|\|_{\lambda\left(\eta_{\mid Y}\right)}$ be the corresponding Quillen metrics on the lines $\lambda\left(\Lambda^{p} E^{*} \otimes\right.$ $\eta), \lambda\left(\eta_{\mid Y}\right)$. We equip the line $\lambda\left(\Lambda E^{*} \otimes \eta\right)$ with the Quillen metric \|\|$_{\lambda\left(\Lambda E^{*} \otimes \eta\right)}$, which is the obvious tensor product of the Quillen metrics \|\|$_{\lambda\left(\Lambda^{p} E^{*} \otimes \eta\right)}$ or their inverses. Similarly, we equip the line $\lambda^{-1}\left(\eta_{\mid Y}\right) \otimes \lambda\left(\Lambda E^{*} \otimes \eta\right)$ with the tensor product of the metrics \|\|$_{\lambda\left(\eta_{\mid Y}\right)}^{-1}$ and \|\|$_{\lambda\left(\Lambda E^{*} \otimes \eta\right)}$.

Recall that the metrics $g^{T X}$ and $g^{T Y}$ are Kähler. 
In [BL1, 2] Bismut and Lebeau calculate $\log \left(\|\sigma\|_{\lambda^{-1}\left(\eta_{\mid Y}\right) \otimes \lambda\left(\Lambda E^{*} \otimes \eta\right)}^{2}\right)$ in terms of explicit local quantities on $X$ or $Y$. This result of [BL1,2] and its relation to our previous work [B2] will be considered in more detail in Sect. 3.

Remark 2.3. The formula established in [BL1,2] is valid for general resolutions of a holomorphic vector bundle on $Y$ by a complex of holomorphic vector bundles on $X$, and not only for resolutions by Koszul complexes.

\section{Equivariant Intersection Over Loop Spaces and a Formula of Bismut-Lebeau}

The purpose of this section is to show that the formula of Bismut-Lebeau [BL1,2] which calculates the Quillen norm of the canonical section $\sigma \in \lambda^{-1}\left(\eta_{\mid Y}\right) \otimes \lambda\left(\Lambda E^{*} \otimes \eta\right)$ can be considered as a formal consequence of an equivariant intersection formula established in [B2, Theorem 3.4]. The analogy is not only in terms of results. In fact a detailed comparison of the intermediary steps and proofs in [BL2] and [B2] shows that they are formally identical.

In particular, we will show that the additive genus $R$ of Gillet and Soule [GS3] is an infinite dimensional version of the genus ${ }^{K} R$ of [B2] evaluated on the infinite dimensional excess normal bundle $\tilde{N}$.

The organization of this section is quite simple. In a)-e), we express formally the various quantities - like the Ray-Singer analytic torsions - whose sum is $\log \left(\|\sigma\|_{\lambda^{-1}\left(\eta_{\mid Y}\right)^{-1} \otimes \lambda\left(\Lambda E^{*} \otimes \eta\right)}^{2}\right)$ in terms of integrals of Bott-Chern currents over loop spaces. We thus discover that $\log \left(\|\sigma\|_{\lambda^{-1}\left(\eta_{\mid Y}\right)^{-1} \otimes \lambda\left(\Lambda E^{*} \otimes \eta\right)}^{2}\right)$ is formally proportional to the left-hand side of the formula of [B2, Theorem 3.4].

In $\mathrm{f}$ ), we calculate formally the right-hand side of the formula of [B2, Theorem 3.4] in terms of well defined Bott-Chern currents on $X$ and $Y$. The genus $R$ appears as a special case of the genus ${ }^{K} R$.

In g), we apply formally the intersection formula of [B2, Theorem 3.4], i.e. we express formally $\log \left(\|\sigma\|_{\lambda^{-1}\left(\eta_{\mid Y}\right) \otimes \lambda\left(\Lambda E^{*} \otimes \eta\right)}^{2}\right)$ as a sum of integrals of Bott-Chern currents
over $X$ and $Y$.

In $h$ ), we state the rigorous formula of Bismut-Lebeau [BL1,2] for $\log \left(\|\sigma\|_{\lambda^{-1}\left(\eta_{\mid Y}\right) \otimes \lambda\left(\Lambda E^{*} \otimes \eta\right)}^{2}\right)$, which turns out to be strictly identical to the formal formula derived in $\mathrm{g}$ ).

Finally in j), we show how the analogy of the results of [BL1,2 and B2] extends to the proofs themselves.

a) An Equivariantly Closed Differential Form on $L X$. We make the same assumptions as in Sect. 2a). Let $\nabla^{\xi}$ be the holomorphic Hermitian connection on $\left(\xi, h^{\xi}\right)$ and let $R^{\xi}$ be its curvature.

Take $x \in L X$. Let $\tau_{t}^{0}$ be the parallel transport operator from $\xi_{x_{0}}$ into $\xi_{x_{t}}$ along the path $s \in[0, t] \rightarrow x_{s}$ with respect to the connection $\nabla^{\xi}$. Set $\tau_{0}^{t}=\left(\tau_{t}^{0}\right)^{-1}$.

For $t \in S_{1}, R_{x_{t}}^{\xi}$ is a 2-form at $x_{t}$ with values in skew-adjoint elements of $\operatorname{End}\left(\xi_{x_{t}}\right)_{t}$. By pulling-back $R_{x_{t}}^{\xi}$ by the map $x \in L X \rightarrow x_{t} \in X$, we will consider $R_{x_{t}}^{\xi}$ is a 2-form at $x \in L X$ with values in skew-adjoint elements of $\operatorname{End}\left(\xi_{x_{t}}\right)$.

Definition 3.1. Let $U_{t}$ be the solution of the differential equation

$$
\frac{d U_{t}}{d t}=U_{t}\left[\tau_{0}^{t} R_{x_{t}}^{\xi} \tau_{t}^{0}\right], \quad U_{0}=I_{\xi_{x_{0}}}
$$


We may expand $U_{1} \tau_{0}^{1}$ in the form

$$
U_{1} \tau_{0}^{1}=\tau_{0}^{1}+\int_{0}^{1} \tau_{0}^{t} R_{x_{t}}^{\xi} \tau_{t}^{0} d t \tau_{0}^{1}+\int_{0 \leq s \leq t \leq 1} \tau_{0}^{s} R_{x_{s}}^{\xi} \tau_{s}^{0} \tau_{0}^{t} R_{x_{t}}^{\xi} \tau_{t}^{0} \tau_{0}^{1} d s d t+\ldots
$$

i.e. $U_{1} \tau_{0}^{1}$ is a sum of forms of degree $0,2,4, \ldots$ with values in $\operatorname{End}\left(\xi_{x_{0}}\right)$.

Definition 3.2.. Set

$$
\beta\left(L \xi, h^{L \xi}\right)=\operatorname{Tr}\left[U_{1} \tau_{0}^{1}\right]
$$

Theorem 3.3. The form $\beta\left(L \xi, h^{L \xi}\right)$ lies in ${ }^{K} P^{L X}$. Moreover

$$
\left(d+i_{K}\right) \beta\left(L \xi, h^{L \xi}\right)=0 .
$$

Proof. Since $R^{\xi}$ is a 2 -form of type $(1,1)$, it is clear that $\beta$ is a sum of forms of type $(p, p)$. The remainder of our theorem is proved in [B4, Theorem 3.9].

Remark 3.3. As pointed out in [B4], the restriction of $\beta\left(L \xi, g^{L \xi}\right)$ to $X$ is given by the form $\operatorname{Tr}\left[\exp \left(R^{\xi}\right)\right]$, i.e. it is a normalized version of the canonical representative of $\operatorname{ch}(\xi)$ in Chern-Weil theory.

Let $\left(\xi^{\prime}, h^{\xi^{\prime}}\right)$ be another holomorphic Hermitian vector bundle on $X$. Let $h^{\xi \otimes \xi^{\prime}}$ be the metric on $\xi \otimes \xi^{\prime}$, which is the tensor product of the metrics $h^{\xi}$ and $h^{\xi^{\prime}}$. Then it is clear that

$$
\beta\left(L\left(\xi \otimes \xi^{\prime}\right), h^{L\left(\xi \otimes \xi^{\prime}\right)}\right)=\beta\left(L \xi, h^{L \xi}\right) \beta\left(L \xi^{\prime}, h^{L \xi^{\prime}}\right) .
$$

b) Accumulating Evidence: A Formal Expression for $\frac{\partial \theta^{\xi}}{\partial s}(0)$. We still make the same assumptions as in Sect. 2a). Recall that $\Lambda\left(T^{*(0,1)} X\right)$ is a $c\left(T_{\mathbb{R}} X\right)$-Clifford module. In fact if $U \in T^{(1,0)} X, V \in T^{(0,1)} X$, if $U^{\prime} \in T^{*(0,1)} X$ corresponds to $U$ by the metric $g^{T X}$, set

$$
c(U)=\sqrt{2} U^{\prime} \wedge, \quad c(V)=-\sqrt{2} i_{V}
$$

We extend by $\mathbb{C}$-linearity the map $c$ to a map $U \in T_{\mathbb{R}} X \otimes_{\mathbb{R}} \mathbb{C}=T^{(1,0)} X \oplus T^{(0,1)} X \rightarrow$ $c(U) \in \operatorname{End}\left(\Lambda\left(T^{*(0,1)} X\right)\right)$. One then easily verifies that if $U, V \in T_{\mathbb{R}} X \otimes_{\mathbb{R}} \mathbb{C}$, then

$$
c(U) c(V)+c(V) c(U)=-2\langle U, V\rangle .
$$

Equation (3.6) shows that indeed $\Lambda\left(T^{*(0,1)} X\right)$ is a $c\left(T_{\mathbb{R}} X\right)$-Clifford module.

Recall that the Kähler form $\omega^{X}$ is associated to the antisymmetric section $\mathbf{J}^{T X}$ of $\operatorname{End}\left(T_{\mathbb{R}} X\right)$ by formula (1.3).

Let $e_{1}, \ldots, e_{2 \operatorname{dim} X}$ be an orthonormal base of $T_{\mathbb{R}} X$.

The following simple result is proved in [BGS2, Proposition 2.4].

Proposition 3.4. The following identity holds

$$
N_{V}^{X}-\frac{1}{2} \operatorname{dim} X=\sqrt{-1} \sum_{i, j} \frac{1}{4} \omega^{X}\left(e_{i}, e_{j}\right) c\left(e_{i}\right) c\left(e_{j}\right) .
$$


By using (3.7) and by proceeding along the lines of [A], [B4, Sect. 2], [B8, Sect. B], we get the following formal formula:

$$
\begin{aligned}
\operatorname{Tr}_{s}\left[\left(N_{V}^{X}-\frac{\operatorname{dim} X}{2}\right) \exp \left(-t \square^{X}\right)\right] \\
=-\left(\frac{i C}{2 \pi}\right)^{\operatorname{dim} X} \int_{L X} \frac{\sqrt{-1} \omega^{L X}}{t} \exp \left(\frac{\bar{\partial}_{K} \partial_{K} \sqrt{-1} \omega^{L X}}{t}\right) \\
\\
\quad \beta\left(L\left(\xi \otimes(\operatorname{det} T X)^{1 / 2}\right), g^{L\left(\xi \otimes(\operatorname{det} T X)^{1 / 2}\right)}\right) .
\end{aligned}
$$

The only minor difference is that in [B4], $C$ was instead the infinite constant $\prod_{1}^{+\infty} k^{2}$. Here we have replaced $\prod_{1}^{+\infty} k^{2}$ by $\prod_{1}^{+\infty}\left(-k^{2}\right)$ by a more careful calculation of the sign of certain expressions which appear in [A, B4, B8]. These expressions are in fact very similar to the right-hand sides of (1.17), (1.18).

Using the notation in [B2, Definition 2.2], we thus find that

$$
\begin{aligned}
\operatorname{Tr}_{s} & {\left[\left(N_{V}^{X}-\frac{\operatorname{dim} X}{2}\right) \exp \left(-t \square^{X}\right)\right] } \\
= & -\left(\frac{i C}{2 \pi}\right)^{\operatorname{dim} X} \frac{i}{2 \pi} \int_{L X} K_{t} \beta\left(L\left(\xi \otimes(\operatorname{det} T X)^{1 / 2}\right), g^{L\left(\xi \otimes(\operatorname{det} T X)^{1 / 2}\right)}\right) .
\end{aligned}
$$

Now recall that if $L X$ was instead a finite dimensional compact complex manifold, by [B8, Proof of Theorem 7], we know the first two terms of the asymptotic expansion of the right-hand side of (3.9) at $t \rightarrow 0$. The first two terms of the asymptotic expansion of the left-hand side of (3.9) have been calculated rigorously in [BGS2, Theorem 2.16]. As explained in [B8], the rigorous formulas obtained in [BGS2, Theorem 2.16] are exactly the formulas one can obtain by extending formally the results of [B8] to the infinite dimensional manifold $L X$. More dramatically, as also explained in [B8], the method used in [BGS2] to derive the rigorous expansion of the left-hand side of (3.9) was found by imitating formally the calculation of the asymptotic expression of the right-hand side of (3.9) in a finite-dimensional situation.

In contrast with these remarkable facts, note that there exists $c>0$ such that as $t \rightarrow+\infty$

$$
\begin{aligned}
\operatorname{Tr}_{s} & {\left[\left(N_{V}^{X}-\frac{\operatorname{dim} X}{2}\right) \exp \left(-t \square^{X}\right)\right] } \\
= & \sum_{0}^{\operatorname{dim} X}(-1)^{p} p \operatorname{dim} H^{p}(X, \xi) \\
& -\frac{\operatorname{dim} X}{2} \sum_{0}^{\operatorname{dim} X}(-1)^{p} \operatorname{dim} H^{p}(X, \xi)+O\left(e^{-c t}\right) .
\end{aligned}
$$

However, if $L X$ was instead finite dimensional and compact, by extending formally the arguments in [B2], we find that as $t \rightarrow+\infty$, in the right-hand side of (3.9), there should be $O\left(\frac{1}{t}\right)$ instead of $O\left(e^{-c t}\right)$. 
Here, there is an unavoidable contradiction. The formal functional integration formalism does not always detect the behaviour of the considered expressions for $t \rightarrow+\infty$, and this for good reasons:

- $L X$ is not compact.

- Certain "irrelevant" terms are neglected when passing from the rigorous expression in terms of the Brownian measure on $L X$ to ill-defined formal integrals of differential forms on $L X$.

Nevertheless, and without fearing the contradiction, in view of (3.9), (3.10), we will write the formal equality

$$
\begin{aligned}
\operatorname{Tr}_{s} & {\left[\left(N_{V}^{X}-\frac{\operatorname{dim} X}{2}\right) \exp \left(-t \square^{X}\right) P^{\perp}\right] } \\
= & -\frac{i}{2 \pi}\left(\frac{i C}{2 \pi}\right)^{\operatorname{dim} X} \int_{L X}{ }^{K} \gamma_{t} \beta\left(L\left(\xi \otimes(\operatorname{det} T X)^{1 / 2}\right), g^{L\left(\xi \otimes(\operatorname{det} T X)^{1 / 2}\right)}\right) .
\end{aligned}
$$

Theorem 3.5. The following formal equality holds

$$
\begin{aligned}
\frac{\partial}{\partial s} \theta_{X}^{\xi}(0)= & \frac{i}{2 \pi}\left(\frac{i C}{2 \pi}\right)^{\operatorname{dim} X} \\
& \int_{L X}{ }^{K} S_{\omega L} \beta\left(L\left(\xi \otimes(\operatorname{det} T X)^{1 / 2}\right), g^{L\left(\xi \otimes(\operatorname{det} T X)^{1 / 2}\right)}\right) .
\end{aligned}
$$

Proof. Using [B2, Eqs. (2.25)-(2.27)], (2.9) and (3.11), (3.12) follows.

c) Accumulating Evidence: A Formal Expression for the Ray-Singer Analytic Torsion of $\Lambda E^{*} \otimes \eta$. We make the same assumptions as in Sect. 3b).

Set for $s \in \mathbb{C}, \operatorname{Re}(s)>\operatorname{dim} X$,

$$
\theta_{X}^{\Lambda E^{*} \otimes \eta}(s)=\sum_{p=0}^{\operatorname{dim} E}(-1)^{p} \theta_{X}^{\Lambda^{p} E^{*} \otimes \eta}(s) .
$$

For $0 \leq p \leq \operatorname{dim} X$, let $g^{\Lambda^{p} E^{*}}$ be the metric induced on $\Lambda^{p} E^{*}$ by the metric $g^{E}$.

Theorem 3.6. The following formal equality holds

$$
\begin{aligned}
\frac{\partial}{\partial s} \theta_{X}^{\Lambda E^{*} \otimes \eta}(0)= & \frac{i}{2 \pi}\left(\frac{i C}{2 \pi}\right)^{\operatorname{dim} X} \int_{L X}{ }^{K} S_{\omega} L X \\
& \beta\left(\sum_{0}^{\operatorname{dim} E}(-1)^{p} \beta\left(L\left(\Lambda^{p} E^{*}\right), g^{L\left(\Lambda^{p} E^{*}\right)}\right)\right) \\
&
\end{aligned}
$$

Proof. Equation (3.14) is a trivial application of Theorem 3.5.

We consider Eq. (3.1) associated to the vector bundle $E$. The operator $U_{1} \tau_{0}^{1}$ then acts on $E_{x_{0}}$. Let $\left(U_{1} \tau_{0}^{1}\right)^{\Lambda E^{*}}$ be the corresponding action of $U_{1} \tau_{0}^{1}$ on $\Lambda E^{*}$. Then

$$
\sum_{0}^{\operatorname{dim} E}(-1)^{p} \beta\left(L\left(\Lambda^{p} E^{*}\right), g^{L\left(\Lambda^{p} E^{*}\right)}\right)=\operatorname{Tr}_{s}\left[\left(U_{1} \tau_{0}^{1}\right)^{\Lambda E^{*}}\right] .
$$




\section{Clearly}

$$
\begin{aligned}
\operatorname{Tr}_{s}\left[\left(U_{1} \tau_{0}^{1}\right)^{\Lambda E^{*}}\right] & =\operatorname{det}\left(1-\left(U_{1} \tau_{0}^{1}\right)^{-1}\right) \\
& =\operatorname{det}\left(\left(U_{1} \tau_{0}^{1}\right)^{1 / 2}-\left(U_{1} \tau_{0}^{1}\right)^{-1 / 2}\right) \operatorname{det}\left(U_{1} \tau_{0}^{1}\right)^{-1 / 2}
\end{aligned}
$$

Also

$$
\operatorname{det}\left(U_{1} \tau_{0}^{1}\right)^{-1 / 2}=\beta\left(L\left((\operatorname{det} E)^{-1 / 2}\right), g^{L\left((\operatorname{det} E)^{-1 / 2}\right)}\right) .
$$

We will proceed as in Atiyah and Witten [A] to give another expression for $\operatorname{det}\left(\left(U_{1} \tau_{0}^{1}\right)^{1 / 2}-\left(U_{1} \tau_{0}^{1}\right)^{-1 / 2}\right)$. Recall that $\frac{D^{E}}{d t}$ is the covariant differentiation operator along the loop $x$ with respect to the connection $\nabla^{E}$. By (1.10), $\frac{D^{E}}{d t}=J^{L E}$, and
so

$$
\frac{D^{E}}{d t}+R^{E}={ }^{K} R^{L E}
$$

We will calculate formally $\operatorname{det}\left(-\frac{{ }^{K} R^{L E}}{2 i \pi}\right)$. First, we determine the formal eigenvalues $\lambda$ of the operator $\frac{D^{E}}{d t}+R^{E}$, i.e. we should find $\lambda \in \mathbb{C}$ and $e \in L E$ nonzero such that

$$
\frac{D^{E} e}{d t}+R^{E} e=\lambda e .
$$

Then by (3.1), (3.19), we get

$$
\frac{d}{d t}\left[U_{t} \tau_{0}^{t} e_{t}\right]=\lambda U_{t} \tau_{0}^{t} e_{t}
$$

From (3.20), we deduce that

$$
U_{t} \tau_{0}^{t} e_{t}=e^{\lambda t} e_{0}
$$

Since $e_{1}=e_{0}$, we get

$$
U_{1} \tau_{0}^{1} e_{0}=e^{\lambda} e_{0}
$$

By (3.22), we see that if $\mu_{1}, \ldots, \mu_{\operatorname{dim} E}$ are the (formal) eigenvalues of $U_{1} \tau_{0}^{1}$, the eigenvalues of the operator ${ }^{K} R^{L E}$ are given by $\mu_{j}+2 i k \pi(1 \leq j \leq \operatorname{dim} E, k \in \mathbb{Z})$. Therefore

$$
\operatorname{det}\left(-\frac{K^{K} R^{L E}}{2 i \pi}\right)=\prod_{1}^{\operatorname{dim} E}\left(-\frac{\mu_{j}}{2 i \pi}\right) \prod_{1}^{+\infty}\left(-\frac{\mu_{j}^{2}}{4 \pi^{2}}-k^{2}\right)
$$

Let $C$ be the infinite constant defined in (1.21). From (1.20), (3.23), we see that

$$
\operatorname{det}\left(-\frac{K R^{L E}}{2 i \pi}\right)=\left(\frac{i C}{2 \pi}\right)^{\operatorname{dim} E} \operatorname{det}\left(\left(U_{1} \tau_{0}^{1}\right)^{1 / 2}-\left(U_{1} \tau_{0}^{1}\right)^{-1 / 2}\right)
$$

In view of (3.15)-(3.17), (3.24), we deduce the following identity:

$$
\begin{aligned}
& \sum_{0}^{\operatorname{dim} E}(-1)^{p} \beta\left(L\left(\Lambda^{p} E^{*}\right), g^{L\left(\Lambda^{p} E^{*}\right)}\right) \\
& \quad=\left(\frac{i C}{2 \pi}\right)^{-\operatorname{dim} E} K c_{\max }\left(L E, g^{L E}\right) \beta\left(L\left((\operatorname{det} E)^{-1 / 2}\right), g^{L\left((\operatorname{det} E)^{-1 / 2}\right)}\right) .
\end{aligned}
$$

Using (3.14) and (3.25), we obtain the following formal result. 
Theorem 3.7. The following formal identity holds

$$
\begin{aligned}
\frac{\partial}{\partial s} \theta_{X}^{\Lambda E^{*} \otimes \eta}(0) & \\
= & \frac{i}{2 \pi}\left(\frac{i C}{2 \pi}\right)^{\operatorname{dim} X-\operatorname{dim} E} \int_{L X}{ }^{K} S_{\omega^{L X}}{ }^{K} c_{\max }\left(L E, g^{L E}\right) \\
& \beta\left(L\left(\eta \otimes(\operatorname{det} T X)^{1 / 2} \otimes(\operatorname{det} E)^{-1 / 2}\right), g^{L\left(\eta \otimes(\operatorname{det} T X)^{1 / 2} \otimes(\operatorname{det} E)^{-1 / 2}\right)}\right) .
\end{aligned}
$$

We finally state an obvious consequence of Theorem 3.5.

Theorem 3.8. The following formal identity holds

$$
\begin{aligned}
\frac{\partial}{\partial s} \theta_{Y}^{\eta_{\mid Y}}(0)= & \frac{i}{2 \pi}\left(\frac{i C}{2 \pi}\right)^{\operatorname{dim} Y} \int_{L X} K_{\omega^{L Y}} \beta\left(L\left(\eta_{\mid Y} \otimes(\operatorname{det} T Y)^{1 / 2}\right),\right. \\
& \left.g^{L\left(\eta_{\mid Y} \otimes(\operatorname{det} T Y)^{1 / 2}\right)}\right) .
\end{aligned}
$$

d) Accumulating Evidence: A Very Formal Expression for $\log \left(|\sigma|_{\lambda^{-1}\left(\eta_{\mid Y}\right) \otimes \lambda\left(\Lambda E^{*} \otimes \eta\right)}^{2}\right)$. Let $N_{H}$ be the operator defining the $\mathbb{Z}$-grading on $\Lambda E^{*}$. Then $N_{H}$ acts on $\Lambda^{p} E^{*}$ by multiplication by $p$.

Let $\mathbb{K}$ be the $\mathbb{Z}$-graded vector space of smooth sections of $\Lambda\left(T^{*(0,1)} X\right) \hat{\otimes} \Lambda E^{*} \otimes \eta$ over $X$. Trace class elements of End( $\mathbb{K})$ have a well-defined supertrace.

Set $v=\sqrt{-1} i_{r}$. Then $v$ acts as an odd operator on $\Lambda\left(T^{*(0,1)} X\right) \otimes \Lambda\left(E^{*}\right)$. Let $v^{*}$ be the adjoint of $v$. The operators $\bar{\partial}^{X}, \bar{\partial}^{X^{*}}, v, v^{*}$ act on $\mathbb{K}$. Set

$$
D^{X}=\bar{\partial}^{X}+\bar{\partial}^{X^{*}}, \quad V=v+v^{*} .
$$

First, we will give a formal expression for

$$
\operatorname{Tr}_{s}\left[\left(N_{H}-\frac{\operatorname{dim} E}{2}\right) \exp \left(-\left(\sqrt{u} D^{X}+\sqrt{T} V\right)^{2}\right)\right] .
$$

Clearly

$$
\begin{aligned}
\operatorname{Tr}_{s} & {\left[\left(N_{H}-\frac{\operatorname{dim} E}{2}\right) \exp \left(-\left(\sqrt{u} D^{X}+\sqrt{T} V\right)^{2}\right)\right] } \\
& =\frac{\partial}{\partial b} \operatorname{Tr}_{s}\left[\exp \left(-\left(\sqrt{u} D^{X}+\sqrt{T} V\right)^{2}+b\left(N_{H}-\frac{\operatorname{dim} E}{2}\right)\right)\right]_{b=0} .
\end{aligned}
$$

We use the notation of Sects. 1b) and 3a). If $f_{1}, \ldots, f_{m}$ is a base of $T_{\mathbb{R}} X$, and if $f^{1}, \ldots, f^{m}$ is the dual base of $T_{\mathbb{R}}^{*} X$, set

$$
\hat{c}\left(\nabla^{E} \sigma\right)=-\sum_{1}^{m} f^{\alpha} \hat{c}\left(\nabla_{f_{\alpha}}^{E} \sigma\right) .
$$

For $b \in \mathbb{R}$, let $H_{t}^{b}$ be the solution of the differential equation

$$
\begin{aligned}
\frac{d H_{t}^{b}}{d t}= & H_{t}^{b}\left[\tau _ { 0 } ^ { t , \Lambda E ^ { * } } \left(R_{x_{t}}^{\Lambda E^{*}}+b\left(N_{H}-\frac{\operatorname{dim} E}{2}\right)\right.\right. \\
& \left.\left.+\sqrt{T} \frac{\hat{c}\left(\nabla^{E} s\left(x_{t}\right)\right)}{\sqrt{2}}-T \frac{\left|s\left(x_{t}\right)\right|^{2}}{2}\right) \tau_{t}^{0, \Lambda E^{*}}\right], \quad H_{0}=I .
\end{aligned}
$$


Then $H_{1}^{b}$ is a form at $x \in L X$ with values in $\operatorname{End}\left(\Lambda E^{*}\right)_{x_{0}}$. Using (3.29) and proceeding as in [B4, Sect. 2c), B8, Sect. B], we see that formally

$$
\begin{aligned}
\operatorname{Tr}_{s} & {\left[\left(N_{H}-\frac{\operatorname{dim} E}{2}\right) \exp \left(-\left(\sqrt{u} D^{X}+\sqrt{T} V\right)^{2}\right)\right] } \\
= & \left(\frac{i C}{2 \pi}\right)^{\operatorname{dim} X} \int_{L X} \exp \left(\frac{\bar{\partial}_{K} \partial_{K} \sqrt{-1} \omega^{L X}}{u}\right) \frac{\partial}{\partial b} \operatorname{Tr}_{s}\left[H_{1}^{b} \tau_{0}^{1, \Lambda E^{*}}\right]_{b=0} \\
& \beta\left(L\left(\eta \otimes(\operatorname{det} T X)^{1 / 2}\right), g^{L\left(\eta \otimes(\operatorname{det} T X)^{1 / 2}\right)}\right) .
\end{aligned}
$$

We will calculate $\frac{\partial}{\partial b} \operatorname{Tr}_{s}\left[H_{1}^{b} \tau_{0}^{1, \Lambda E^{*}}\right]_{b=0}$. Let $H_{t}^{\prime b}$ be the solution of the differential equation

$$
\frac{d H_{t}^{\prime} b}{d t}=H_{t}^{\prime b}\left[\tau_{0}^{t, \Lambda E^{*}}\left(R_{x_{t}}^{\Lambda E^{*}}+b N_{H}+\sqrt{T} \frac{\hat{c}\left(\nabla^{E} s\left(x_{t}\right)\right.}{\sqrt{2}}\right) \tau_{t}^{0, \Lambda E^{*}}\right], \quad H_{0}^{\prime}=I .
$$

Then

$$
H_{1}^{b}=H_{1}^{\prime b} \exp \left\{-T \int_{0}^{1} \frac{\mid s\left(\left.x_{t}\right|^{2}\right.}{2} d t-b \frac{\operatorname{dim} E}{2}\right\} .
$$

If $b \in \mathbb{R}$, let $U_{t}^{b}$ be the solution of the differential equation

$$
\frac{d U_{t}^{b}}{d t}=U_{t}^{b}\left[\tau_{0}^{t}\left(R_{x_{t}}^{E}-b\right) \tau_{t}^{0}\right], \quad U_{0}=I
$$

Then $U_{t}^{b}$ is a form at $x \in L X$ with values in $\operatorname{End}\left(E_{x_{0}}\right)$. An easy adaptation of a formula proved in [B7, Theorem 5.1] and $(3.33),(3,34)$ show that

$$
\begin{aligned}
\operatorname{Tr}_{s}\left[H_{1}^{b} \tau_{0}^{1, \Lambda E^{*}}\right]= & \operatorname{Tr}_{s}\left[\left(U_{1}^{b} \tau_{0}^{1}\right)^{\Lambda E^{*}}\right] \exp \left\{-\frac{T}{2}\left(\int_{0}^{1}\left|s\left(x_{t}\right)\right|^{2} d t\right.\right. \\
& \left.\left.+\left\langle\nabla^{E} s,\left(\frac{D}{D t}+R^{L E}+b i \mathbf{J}^{L E}\right)^{-1} \nabla^{E} s\right\rangle\right)-\frac{b \operatorname{dim} E}{2}\right\} .
\end{aligned}
$$

Note that (3.36) is a completely rigorous formula.

Also by proceeding as in (3.15)-(3.25), we get

$$
\begin{aligned}
\operatorname{Tr}_{s}\left[\left(U_{1}^{b} \tau_{0}^{1}\right)^{\Lambda E^{*}}\right]= & \left(\frac{i C}{2 \pi}\right)^{-\operatorname{dim} E} \operatorname{det}\left(-\left(\frac{{ }^{K} R^{L E}-b}{2 i \pi}\right)\right) \\
& \beta\left(L\left((\operatorname{det} E)^{-1 / 2}\right), g^{L\left((\operatorname{det} E)^{-1 / 2}\right)}\right) e^{b \operatorname{dim} E / 2} .
\end{aligned}
$$

Using (3.36), (3.37), we obtain

$$
\begin{aligned}
& \frac{\partial}{\partial b} \operatorname{Tr}_{s}\left[H_{1}^{b} \tau_{0}^{1, \Lambda E^{*}}\right]_{b=0} \\
& \quad=\frac{i}{2 \pi}\left(\frac{i C}{2 \pi}\right)^{-\operatorname{dim} E} s^{*}\left({ }^{K} c_{T}\left(L E, g^{L E}\right)\right) \beta\left(L(\operatorname{det} E)^{-1 / 2}, g^{L(\operatorname{det} E)^{-1 / 2}}\right) .
\end{aligned}
$$

From (3.32), (3.38), we finally obtain the key result. 
Theorem 3.9. For $u>0, T \geq 0$, the following formal identity holds

$$
\begin{aligned}
\operatorname{Tr}_{s} & {\left[\left(N_{H}-\frac{\operatorname{dim} E}{2}\right) \exp \left(-\left(\sqrt{u} D^{X}+\sqrt{T} V\right)^{2}\right)\right] } \\
= & \left(\frac{i C}{2 \pi}\right)^{\operatorname{dim} X-\operatorname{dim} E} \frac{i}{2 \pi} \int_{L X} K \alpha_{u} s^{*}\left({ }^{K} c_{T}\left(L E, g^{L E}\right)\right) \\
& \beta\left(L\left(\eta \otimes(\operatorname{det} T X)^{1 / 2} \otimes(\operatorname{det} E)^{-1 / 2}\right), g^{L\left(\eta \otimes(\operatorname{det} T X)^{1 / 2} \otimes(\operatorname{det} E)^{-1 / 2}\right)}\right) .
\end{aligned}
$$

Let $P^{\operatorname{Ker} D^{X}}$ be the orthogonal projection from $\mathbb{K}$ on $\operatorname{Ker} D^{X}$.

Theorem 3.10. For any $T \geq 0$, the following identity holds

$$
\begin{aligned}
& \lim _{u \rightarrow+\infty} \operatorname{Tr}_{s}\left[\left(N_{H}-\frac{\operatorname{dim} E}{2}\right) \exp \left(-\left(\sqrt{u} D^{X}+\sqrt{T} V\right)^{2}\right)\right] \\
& =\operatorname{Tr}_{s}\left[P^{\operatorname{Ker} D^{X}}\left(N_{H}-\frac{\operatorname{dim} E}{2}\right) \exp \left(-T\left(P^{\operatorname{Ker} D^{X}} V P^{\operatorname{Ker} D^{X}}\right)^{2}\right)\right]
\end{aligned}
$$

Proof. By proceeding as in Bismut-Lebeau [BL2, Proof of Theorem 8.2], we easily obtain (3.40).

Let $H\left(\Lambda E^{*} \otimes \eta\right)$ be the cohomology groups of the sheaf $\mathscr{O}_{X}\left(\Lambda E^{*} \otimes \eta\right)$. By Hodge theory, we know that

$$
\operatorname{Ker} D^{X} \cong H\left(\Lambda E^{*} \otimes \eta\right)
$$

Also $v=\sqrt{-1} i_{r}$ acts naturally on $H\left(\Lambda E^{*} \otimes \eta\right)$. The operator $P^{\operatorname{Ker} D^{X}} v P^{\operatorname{Ker} D^{X}}$ represents this natural action of $v$ on $H\left(\Lambda E^{*} \otimes \eta\right)$ under the isomorphism (3.41), and $P^{\operatorname{Ker} D^{X}} v^{*} P^{\operatorname{Ker} D^{X}}$ is the adjoint of $P^{\operatorname{Ker} D^{X}} v P^{\operatorname{Ker} D^{X}}$ with respect to the metric induced by the $L_{2}$ metric (2.4) on $\operatorname{Ker} D^{X}$.

We now assume that the complex $\left(\mathbb{K}, \bar{\partial}^{X}+v\right)$ is acyclic, and that the corresponding spectral sequence degenerates at $E_{2}$, i.e. $\left(H\left(\Lambda E^{*} \otimes \eta\right), v\right)$ is an acyclic complex. Then $P^{\operatorname{Ker} D^{X}} V P^{\operatorname{Ker} D^{X}}$ is an invertible map. Also by [BL2, Theorem 1.7], the sheaf $Q_{Y}\left(\eta_{\mid Y}\right)$ has zero cohomology.

For $s \in \mathbb{C}, \operatorname{Re}(s)>0$, set

$$
\begin{aligned}
\delta(s)= & \frac{1}{\Gamma(s)} \int_{0}^{+\infty} T^{s-1} \\
& \operatorname{Tr}_{s}\left[P^{\operatorname{Ker} D^{X}}\left(N_{H}-\frac{\operatorname{dim} E}{2}\right) \exp \left(-T\left(P^{\operatorname{Ker} D^{X}} V P^{\operatorname{Ker} D^{X}}\right)^{2}\right)\right] d t .
\end{aligned}
$$

Then $\delta(s)$ extends to a meromorphic function, which is holomorphic at $s=0$.

For $0 \leq p \leq \operatorname{dim} X$, let $T^{p}(v)$ be the canonical section of $\bigotimes_{i=0}^{\operatorname{dim} E}\left(\operatorname{det} H^{p}\left(\Lambda^{i} E^{*} \otimes\right.\right.$ $\eta))^{(-1)^{i}}$ associated to the acyclic complex $\left(H^{p}\left(\Lambda E^{*} \otimes \eta\right), v\right)$, whose inverse is constructed in [BGS1, Definition 1.1]. Set

$$
T(v)=\bigotimes_{p=0}^{\operatorname{dim} E}\left(T^{p}(v)\right)^{(-1)^{p+1}}
$$


Then $T(v)$ is a nonzero section of $\lambda(\xi)$. By [BGS1, Proposition 1.5], we know that

$$
\log \left(|T(v)|_{\lambda(\xi)}^{2}\right)=-\delta^{\prime}(0)
$$

Equivalently

$$
\log \left(|\sigma|_{\lambda^{-1}\left(\eta_{\mid Y}\right) \otimes \lambda\left(\Lambda E^{*} \otimes \eta\right)}^{2}\right)=-\delta^{\prime}(0)
$$

Now formally

$$
\begin{gathered}
\lim _{u \rightarrow+\infty} \frac{i}{2 \pi}\left(\frac{i C}{2 \pi}\right)^{\operatorname{dim} X-\operatorname{dim} E} \int_{L X} \exp \left(\frac{\bar{\partial}_{K} \partial_{K} \sqrt{-1} \omega^{L X}}{u}\right) s^{*}\left({ }^{K} c_{T}\left(L E, g^{L E}\right)\right) \\
\beta\left(L(\eta \otimes \operatorname{det} T X)^{1 / 2} \otimes(\operatorname{det} E)^{-1 / 2}, g^{L\left(\eta \otimes(\operatorname{det} T X)^{1 / 2} \otimes(\operatorname{det} E)^{-1 / 2}\right)}\right) \\
=\frac{i}{2 \pi}\left(\frac{i C}{2 \pi}\right)^{\operatorname{dim} X-\operatorname{dim} E} \int_{L X} s^{*}\left({ }^{K} c_{T}\left(L E, g^{L E}\right)\right) \\
\left.\beta\left(L\left(\eta \otimes(\operatorname{det} T X)^{1 / 2}\right) \otimes(\operatorname{det} E)^{-1 / 2}\right), g^{L\left(\eta \otimes(\operatorname{det} T X)^{1 / 2} \otimes(\operatorname{det} E)^{-1 / 2}\right)}\right) .
\end{gathered}
$$

In view of [B2, Definitions 2.19 and 2.20] and of (3.39)-(3.45), we therefore easily obtain:

Theorem 3.11. The following formal equality holds

$$
\begin{aligned}
\log \left(|\sigma|_{\lambda^{-1}\left(\eta_{\mid Y}\right) \otimes \lambda\left(\Lambda E^{*} \otimes \eta\right)}^{2}\right)= & -\frac{i}{2 \pi}\left(\frac{i C}{2 \pi}\right)^{\operatorname{dim} X-\operatorname{dim} E} \int_{L X} K^{L X} \tilde{e}^{L X}\left(L E, g^{L E}\right) \\
& \beta\left(L\left(\eta \otimes(\operatorname{det} T X)^{1 / 2} \otimes(\operatorname{det} E)^{-1 / 2}\right)\right. \\
& \left.g^{L\left(\eta \otimes(\operatorname{det} T X)^{1 / 2} \otimes(\operatorname{det} E)^{-1 / 2}\right)}\right) .
\end{aligned}
$$

In the sequel, we will do as if the formal equality (3.46) was valid in full generality. e) Accumulating Evidence: A Formal Expression for $\log \left(\|\sigma\|_{\lambda^{-1}\left(\eta_{\mid Y}\right) \otimes \lambda\left(\Lambda E^{*} \otimes \eta\right)}^{2}\right)$. We now establish a key formal identity.

Theorem 3.12. The following formal identity holds:

$$
\begin{aligned}
& \log \left(\|\sigma\|_{\lambda^{-1}\left(\eta_{\mid Y}\right) \otimes \lambda\left(\Lambda E^{*} \otimes \eta\right)}^{2}\right) \\
& =\frac{i}{2 \pi}\left(\frac{i C}{2 \pi}\right)^{\operatorname{dim} X-\operatorname{dim} E}\left[-\int_{L X} K_{\tilde{e}^{L X}}\left(L E, g^{L E}\right)\right. \\
& \beta\left(L\left(\eta \otimes(\operatorname{det} T X)^{1 / 2} \otimes(\operatorname{det} E)^{-1 / 2}\right), g^{L\left(\eta \otimes(\operatorname{det} T X)^{1 / 2} \otimes(\operatorname{det} E)^{-1 / 2}\right)}\right) \\
& -\int_{L X}{ }^{K} S_{\omega} L X{ }^{K} c_{\max }\left(L E, g^{L E}\right) \\
& \beta\left(L\left(\eta \otimes(\operatorname{det} T X)^{1 / 2} \otimes(\operatorname{det} E)^{-1 / 2}\right), g^{L\left(\eta \otimes(\operatorname{det} T X)^{1 / 2} \otimes(\operatorname{det} E)^{-1 / 2}\right)}\right) \\
& \left.+\int_{L Y}{ }^{K} S_{\omega^{L Y}} \beta\left(L\left(\eta_{\mid Y} \otimes(\operatorname{det} T Y)^{1 / 2}\right), g^{L\left(\eta_{\mid Y} \otimes(\operatorname{det} T Y)^{1 / 2}\right)}\right)\right] .
\end{aligned}
$$

Proof. Using (2.10), (3.26), (3.27), (3.46) and the fact that $\operatorname{dim} X-\operatorname{dim} E=\operatorname{dim} Y$, we get (3.47). 
Remark 3.13. Observe that the identification of line bundles (2.1) also identifies the metrics. Set

$$
\mu=\beta\left(L\left(\eta \otimes(\operatorname{det} T X)^{1 / 2} \otimes(\operatorname{det} E)^{-1 / 2}\right), g^{L\left(\eta \otimes(\operatorname{det} T X)^{1 / 2} \otimes(\operatorname{det} E)^{-1 / 2}\right)}\right) .
$$

Then, the restriction of $\mu$ to $L Y$ is exactly the form $\beta\left(L\left(\eta_{\mid Y} \otimes(\operatorname{det} T Y)^{1 / 2}\right)\right.$, $\left.g^{L\left(\eta_{\mid Y} \otimes(\operatorname{det} T Y)^{1 / 2}\right)}\right)$.

Moreover by Theorem 3.3, $\mu$ is a sum of forms of type $(p, p)$, and also

$$
\partial_{K} \mu=0, \quad \bar{\partial}_{K} \mu=0 .
$$

Therefore the right-hand side of (3.47) is formally proportional to the left-hand side of the identity in [B2, Eqs. (3.10), (3.11)], with $\mu$ given by (3.48). The idea will now be to calculate formally the objects which appear in the right-hand side of [B2, Eqs. (3.10), (3.11)].

f) A Formal Expression for Certain Finite Dimensional Integrals. Recall that if $B$ is a $(q, q)$ matrix, then

$$
\operatorname{Td}(B)=\operatorname{det}\left(\frac{B}{1-e^{-B}}\right) .
$$

Theorem 3.14. The following formal identity holds:

$$
\begin{gathered}
\frac{i}{2 \pi}\left(\frac{i C}{2 \pi}\right)^{\operatorname{dim} X-\operatorname{dim} E}\left\{-\int_{X} K c_{\max }^{-1}\left(N_{X / L X}, g^{N_{X / L X}}\right)^{K} \tilde{e}^{X}\left(L E, g^{L E}\right)\right. \\
\left.\beta\left(L\left(\eta \otimes(\operatorname{det} T X)^{1 / 2} \otimes(\operatorname{det} E)^{-1 / 2}\right), g^{L\left(\eta \otimes(\operatorname{det} T X)^{1 / 2} \otimes(\operatorname{det} E)^{-1 / 2}\right)}\right)\right\} \\
=-\int_{X} \operatorname{Td}\left(T X, g^{T X}\right) T^{X}\left(\Lambda E^{*}, g^{\Lambda E^{*}}\right) \operatorname{ch}\left(\eta, g^{\eta}\right) .
\end{gathered}
$$

Proof. By Proposition 1.1 and Theorem 1.8, we see that the left-hand side of (3.50) is given by

$$
\begin{aligned}
& -\frac{i}{2 \pi}\left(\frac{i C}{2 \pi}\right)^{\operatorname{dim} X-\operatorname{dim} E} \int_{X} C^{-\operatorname{dim} X} \hat{A}\left(R^{T X}\right)\left(\frac{C}{2 \pi i}\right)^{\operatorname{dim} E} 2 \pi i \varphi^{-1}\left(\tilde{T}^{X}\left(\Lambda E^{*}, g^{\Lambda E^{*}}\right)\right) \\
& e^{-\operatorname{Tr}\left[R^{E}\right]+1 / 2 \operatorname{Tr}\left[R^{T X}\right]} \operatorname{Tr}\left[\exp \left(R^{\eta}\right)\right] .
\end{aligned}
$$

Using Theorem 1.6 and the fact that $\tilde{e}\left(E, g^{E}\right)$ has total degree $2 \operatorname{dim} E-2$, we see that (3.51) is equal to

$$
\begin{aligned}
& \left(\frac{i}{2 \pi}\right)^{\operatorname{dim} X} \int_{X} \hat{A}\left(R^{T X}\right) e^{1 / 2 \operatorname{Tr}\left[R^{T X}\right]} \operatorname{det}\left(\frac{1-e^{R^{E}}}{-R^{E}}\right) \\
& \quad e^{-\operatorname{Tr}\left[R^{E}\right]}(-1)^{\operatorname{dim} E}(2 i \pi)^{\operatorname{dim} E-1} s^{*} \tilde{e}\left(E, g^{E}\right) \operatorname{Tr}\left[\exp \left(R^{\eta}\right)\right] \\
& =\left(\frac{i}{2 \pi}\right)^{\operatorname{dim} X} \int_{X} \operatorname{Td}\left(R^{T X}\right) \operatorname{Td}^{-1}\left(R^{E}\right)(-1)^{\operatorname{dim} E}(2 i \pi)^{\operatorname{dim} E-1} \\
& s^{*} \tilde{e}\left(E, g^{E}\right) \operatorname{Tr}\left[\exp \left(R^{\eta}\right)\right] \\
& =-\int_{X} \operatorname{Td}\left(T X, g^{T X}\right) \operatorname{Td}^{-1}\left(E, g^{E}\right) s^{*} \tilde{e}\left(E, g^{E}\right) \operatorname{ch}\left(\eta, g^{\eta}\right) .
\end{aligned}
$$

By using again Theorem 1.6 again, we get (3.50). 
Recall that $P^{Y}$ is the vector space of smooth forms on $Y$ which are sums of forms of type $(p, p)$. Also $P^{Y, 0}$ is the set of $\alpha \in P^{Y}$ such that there exist smooth forms $\beta$, $\gamma$ on $Y$ for which $\alpha=\partial \beta+\bar{\partial} \gamma$.

Let $\widetilde{\mathrm{Td}}\left(T Y, T X_{\mid Y}, g^{T X_{\mid Y}}\right)$ be the Bott-Chern class in $P^{Y} / P^{Y, 0}$ defined in [BGS1, Sect. 1f)] which is associated to the exact sequence of holomorphic Hermitian vector bundles $0 \rightarrow T Y \rightarrow T X_{\mid Y} \rightarrow N_{X / Y} \rightarrow 0$, and is such that

$$
\begin{aligned}
\frac{\bar{\partial} \partial}{2 i \pi} \widetilde{\mathrm{Td}}\left(T Y, T X_{\mid Y}, g^{T X_{\mid Y}}\right)= & \operatorname{Td}\left(T X_{\mid Y}, g^{T X_{\mid Y}}\right) \\
& -\operatorname{Td}\left(T Y, g^{T Y}\right) T d\left(N_{X / Y}, g^{N_{X / Y}}\right) .
\end{aligned}
$$

The class $\widetilde{\operatorname{Td}}\left(T Y, T X_{\mid Y}, g^{T X_{\mid Y}}\right)$ is normalized by the fact that if the considered exact sequence splits holomorphically and metrically, then it vanishes in $P^{Y} / P^{Y, 0}$.

Theorem 3.15. The following formal identity holds:

$$
\begin{gathered}
\frac{i}{2 \pi}\left(\frac{i C}{2 \pi}\right)^{\operatorname{dim} X-\operatorname{dim} E} \int_{Y}{ }^{K} c_{\max }\left(\tilde{N}, g^{\tilde{N}}\right)^{\widetilde{K} c_{\max }^{-1}}\left(N_{Y / L Y}, N_{X / L X_{\mid Y}}, g^{N_{X / L Y} Y_{\mid Y}}\right) \\
\beta\left(L\left(\eta \otimes(\operatorname{det} T X)^{1 / 2} \otimes(\operatorname{det} E)^{-1 / 2}\right), g^{L\left(\eta \otimes(\operatorname{det} T X)^{1 / 2} \otimes(\operatorname{det} E)^{-1 / 2}\right)}\right) \\
=\int_{Y} \operatorname{Td}^{-1}\left(N_{Y / X}, g^{\left.N_{Y / X}\right)} \widetilde{\mathrm{Td}}\left(T Y, T X_{\mid Y}, g^{T X_{\mid Y}}\right) \operatorname{ch}\left(\eta_{\mid Y}, g^{\eta_{\mid Y}}\right)\right.
\end{gathered}
$$

Proof. Let $\tilde{\hat{A}}\left(T Y, T X_{\mid Y}, g^{T X_{\mid Y}}\right)$ be the Bott-Chern class in $P^{Y} / P^{Y, 0}$ associated to the exact sequence $0 \rightarrow T Y \rightarrow T X_{\mid Y} \rightarrow N_{X / Y} \rightarrow 0$ and the complex Hirzebruch $\hat{A}$ genus defined in (1.19). By [B2, Eq. (1.28)] and by Proposition 1.1, we get

$$
\begin{aligned}
& \frac{\bar{\partial} \partial}{2 i \pi} \widetilde{K^{c-1}}\left(N_{Y / L Y}, N_{X / L X_{\mid Y}}, g^{N_{X / L X} \mid Y}\right) \\
& \quad=C^{-\operatorname{dim} X}\left(\hat{A}\left(R^{T X}\right)-\hat{A}\left(R^{T Y}\right) \hat{A}\left(R^{N_{Y / X}}\right)\right), \\
& \frac{\bar{\partial} \partial}{2 i \pi} \tilde{\hat{A}}\left(T Y, T X_{\mid Y}, g^{T X_{\mid Y}}\right) \\
& \quad=\hat{A}\left(\frac{-R^{T X}}{2 i \pi}\right)-\hat{A}\left(\frac{-R^{T Y}}{2 i \pi}\right) \hat{A}\left(\frac{-R^{N_{Y / X}}}{2 i \pi}\right) .
\end{aligned}
$$

Using the fact that $\hat{A}(x)$ is an even function of $x$, (3.55) and the uniqueness of Bott-Chern classes [BGS1, Theorem 1.29], we get

$$
\begin{aligned}
& \widetilde{{ }^{K} c_{\max }^{-1}}\left(N_{Y / L Y}, N_{X / L X}, g^{N_{X / L X_{\mid Y}}}\right) \\
& \quad=2 i \pi C^{-\operatorname{dim} X} \varphi^{-1} \tilde{\hat{A}}\left(T Y, T X_{\mid Y}, g^{T X_{\mid Y}}\right) \text { in } P^{Y} / P^{Y, 0} .
\end{aligned}
$$

Also since $\hat{A}(x)$ is an even function, it is clear from (3.55) that $\tilde{\hat{A}}\left(T Y, T X_{\mid Y}, g^{T X_{\mid Y}}\right)$ is a class of sums of forms of degree $4 p+2$. Using an analogue of Proposition 1.1 for $\tilde{N}$ and (3.56), we thus find that the left-hand side of (3.54) is exactly

$$
\int_{Y} \operatorname{Td}^{-1}\left(N_{Y / X}, g^{N_{Y / X}}\right) \tilde{\hat{A}}\left(T Y, T X_{\mid Y}, g^{T X_{\mid Y}}\right) e^{-\frac{\operatorname{Tr} r R^{T X}}{2 i \pi}} \operatorname{ch}\left(\eta_{\mid Y}, g^{\eta_{\mid Y}}\right) .
$$


Recall that the isomorphism (2.1) also identifies the metrics. So we find that

$$
\tilde{\hat{A}}\left(T Y, T X_{\mid Y}, g^{T X_{\mid Y}}\right) e^{-\frac{\operatorname{Tr}\left[R^{T X}\right]}{2 i \pi}}=\widetilde{\mathrm{Td}}\left(T Y, T X_{\mid Y}, g^{T X_{\mid Y}}\right) \quad \text { in } \quad P^{Y} / P^{Y, 0} \text {. }
$$

Using (3.57), (3.58), we get (3.54).

Recall that the genus ${ }^{K} R$ was defined in [B2, Definition 1.20)]. In the right-hand side of [B2, Eq. (3.10)], the class ${ }^{K} R(\tilde{N})$ appears. We will now calculate this class in our special situation.

Let $\zeta(s)$ be the Riemann zeta function. We introduce the genus $R$ of Gillet and Soulé [GS3].

Definition 3.16. Let $R(x)$ be the formal power series

$$
R(x)=\sum_{\substack{n \geq 1 \\ n \text { odd }}}\left(2 \frac{\zeta^{\prime}(-n)}{\zeta(-n)}+\sum_{j=1}^{n} \frac{1}{j}\right) \zeta(-n) \frac{x^{n}}{n !} .
$$

We identify $R$ with the corresponding additive genus. In particular $R\left(N_{Y / X}\right.$, $g^{N_{Y / X}}$ ) lies in $P^{Y}$.

Let $\tilde{\varphi}$ be the homomorphism of $\Lambda^{\text {even }}\left(T_{\mathbb{R}}^{*} X\right)$ into itself which maps $\alpha \in \Lambda^{2 p}\left(T_{\mathbb{R}}^{*} X\right)$ into $(-2 i \pi)^{-p} \alpha \in \Lambda^{2 p}\left(T_{\mathbb{R}}^{*} X\right)$.

Theorem 3.17. The following formal identity of differential forms holds on $Y$ :

$$
\frac{i}{2 \pi} \tilde{\varphi}^{K} R\left(\tilde{N}, g^{\tilde{N}}\right)=R\left(N_{Y / X}, g^{N_{Y / X}}\right) .
$$

Proof. The spectrum of the operator $J^{\tilde{N}}$ is exactly $\{2 i k \pi\}_{k \in \mathbb{Z}^{*}}$. Using the notation in [B2, Proposition 1.24], we see that

$$
{ }^{K} R\left(\tilde{N}, g^{\tilde{N}}\right)=\sum_{k \in \mathbb{Z}^{*}} R^{k}\left(-\frac{R^{N_{Y / X}}}{2 i \pi}\right) .
$$

Now

$$
R^{-\mu}(x)=-R^{\mu}(-x)
$$

We thus rewrite (3.61) in the form

$$
{ }^{K} R\left(\tilde{N}, g^{\tilde{N}}\right)=\sum_{k \in N^{*}}\left(R^{k}\left(-\frac{R^{N_{Y / X}}}{2 i \pi}\right)-R^{k}\left(\frac{R^{N_{Y / X}}}{2 i \pi}\right)\right) .
$$

Using (3.63), it is clear that to evaluate (3.60), we should calculate

$$
\frac{i}{2 \pi} \sum_{k \in \mathbb{N}^{*}}\left(R^{k}\left(\frac{i x}{2 \pi}\right)-R^{k}\left(-\frac{i x}{2 \pi}\right)\right) \text {. }
$$

Now for $k \in \mathbb{N}^{*}$, by [B2, Definition 1.22], we get

$$
\begin{aligned}
\frac{i}{2 \pi} R^{k}\left(\frac{i x}{2 \pi}\right)= & i\left(2 \Gamma^{\prime}(1)-2 \log (2 k \pi)-\log \left(1+\frac{i x}{2 k \pi}\right)\right) \\
& \left(2 k \pi\left(1+\frac{i x}{2 k \pi}\right)\right)^{-1} .
\end{aligned}
$$


Set

$$
\begin{gathered}
h_{k}(x)=i\left(\Gamma^{\prime}(1)-2 \log (2 k \pi)-\log \left(1+\frac{i x}{2 k \pi}\right)\right)\left(2 k \pi\left(1+\frac{i x}{2 k \pi}\right)\right)^{-1} \\
m_{k}(x)=i \Gamma^{\prime}(1)\left(2 k \pi\left(1+\frac{i x}{2 k \pi}\right)\right)^{-1}
\end{gathered}
$$

By (3.64), (3.65), we get

$$
\frac{i}{2 \pi} R_{k}\left(\frac{i x}{2 \pi}\right)=h_{k}(x)+m_{k}(x) .
$$

Now using Bismut [B7, Eqs. (8.37)-(8.39)], Bismut and Soulé [B7, Appendix, Theorem 1 and Eqs. (10), (11)], and also (3.66), we get

$$
\frac{i}{2 \pi} \sum_{k \in \mathbb{N}^{*}}\left(R^{k}\left(\frac{i x}{2 i \pi}\right)-R^{k}\left(-\frac{i x}{2 i \pi}\right)\right)=R(x) .
$$

Equation (3.60) follows from (3.63), (3.67).

Remark 3.18. It is very important to observe that the only step where we needed to convert the ill-defined ${ }^{K} R\left(\tilde{N}, g^{\tilde{N}}\right)$ into a well-defined expression is Eq. (3.63), which ensures the convergence of the ill defined series (3.61).

Theorem 3.19. The following formal identity holds:

$$
\begin{aligned}
- & \frac{i}{2 \pi}\left(\frac{i C}{2 \pi}\right)^{\operatorname{dim} X-\operatorname{dim} E} \int_{Y} K_{c_{\max }^{-1}\left(N_{Y / L Y}\right)^{K} R(\tilde{N})} \\
& \beta\left(L\left(\eta \otimes(\operatorname{det} T X)^{1 / 2} \otimes(\operatorname{det} E)^{-1 / 2}\right), g^{L\left(\eta \otimes(\operatorname{det} T X)^{1 / 2} \otimes(\operatorname{det} E)^{-1 / 2}\right)}\right) \\
= & -\int_{Y} \operatorname{Td}(T Y) R\left(N_{Y / X}\right) \operatorname{ch}(\eta) .
\end{aligned}
$$

Proof. By using (1.22) and (3.60), (3.68) follows.

g) A Formal Formula for $\log \left(\|\sigma\|_{\lambda^{-1}\left(\eta_{\mid Y}\right) \otimes \lambda\left(\Lambda E^{*} \otimes \eta\right)}^{2}\right)$ in Terms of Finite Dimensional Integrals. The main formal result of this paper is as follows.

Theorem 3.20. The following formal identities hold:

$$
\begin{aligned}
\log \left(\|\sigma\|_{\lambda^{-1}\left(\eta_{\mid Y}\right) \otimes \lambda\left(\Lambda E^{*} \otimes \eta\right)}^{2}\right) & \\
= & -\int_{X} \operatorname{Td}\left(T X, g^{T X}\right) T^{X}\left(\Lambda E^{*}, g^{\Lambda E^{*}}\right) \operatorname{ch}\left(\eta, g^{\eta}\right) \\
& +\int_{Y} \operatorname{Td}^{-1}\left(N_{Y / X}, g^{N_{Y / X}}\right) \widetilde{\operatorname{Td}}\left(T Y, T X_{\mid Y}, g^{T X_{\mid Y}}\right) \operatorname{ch}\left(\eta_{\mid Y}, g^{\eta_{\mid Y}}\right) \\
& -\int_{Y} \operatorname{Td}(T Y) R\left(N_{Y / X}\right) \operatorname{ch}\left(\eta_{\mid Y}\right),
\end{aligned}
$$




$$
\begin{aligned}
\log \left(\|\sigma\|_{\lambda^{-1}\left(\eta_{\mid Y}\right) \otimes \lambda\left(\Lambda E^{*} \otimes \eta\right)}^{2}\right) & \\
= & -\int_{X} \operatorname{Td}\left(T X, g^{T X}\right) T^{X}\left(\Lambda E^{*}, g^{\Lambda E^{*}}\right) \operatorname{ch}\left(\eta, g^{\eta}\right) \\
& +\int_{Y} \operatorname{Td}^{-1}\left(N_{Y / X}, g^{\left.N_{Y / X}\right)} \widetilde{\operatorname{Td}}\left(T Y, T X_{\mid Y}, g^{T X_{\mid Y}}\right) \operatorname{ch}\left(\eta_{\mid Y}, g^{\eta_{\mid Y}}\right)\right. \\
& -\int_{X} \operatorname{Td}(T X) R(T X) \operatorname{ch}\left(\Lambda E^{*} \otimes \eta\right)+\int_{Y} \operatorname{Td}(T Y) R(T Y) \operatorname{ch}\left(\eta_{\mid Y}\right) .
\end{aligned}
$$

Proof. The first identity in (3.69) follows from the rigorous first identity in [B2, Theorem 3.4] applied to the right-hand side of (3.47) in Theorem 3.12 and from Theorems 3.14, 3.15, 3.19. Using [BGS4, Theorem 2.5], the second identity in (3.69) follows easily from the first one. One may instead use the second identity in [B2, Theorem 3.4].

Remark 3.21. It is essential to remark at this stage that both sides of the identities (3.69) are well-defined and are not "formal" objects. Only the identities (3.69) are formal (for the moment) since the identities which connect the two sides are themselves formal.

h) A Theorem of Bismut-Lebeau. The following result is the main result of BismutLebeau [BL1], [BL2, Theorem 0.1].

Theorem 3.22. The following identities hold

$$
\begin{aligned}
& \log \left(\|\sigma\|_{\lambda^{-1}\left(\eta_{\mid Y}\right) \otimes \lambda\left(\Lambda E^{*} \otimes \eta\right)}^{2}\right) \\
&=-\int_{X} \operatorname{Td}\left(T X, g^{T X}\right) T^{X}\left(\Lambda E^{*}, g^{\Lambda E^{*}}\right) \operatorname{ch}\left(\eta, g^{\eta}\right) \\
&+\int_{Y} \operatorname{Td}^{-1}\left(N_{Y / X}, g^{N_{Y / X}}\right) \widetilde{\mathrm{Td}}\left(T Y, T X_{\mid Y}, g^{T X_{\mid Y}}\right) \operatorname{ch}\left(\eta_{\mid Y}, g^{\eta_{\mid Y}}\right) \\
&-\int_{Y} \operatorname{Td}(T Y) R\left(N_{Y / X}\right) \operatorname{ch}\left(\eta_{\mid Y}\right) \\
& \log \left(\|\sigma\|_{\lambda^{-1}\left(\eta_{\mid Y}\right) \otimes \lambda\left(\Lambda E^{*} \otimes \eta\right)}^{2}\right) \\
&=-\int_{X} \operatorname{Td}\left(T X, g^{T X}\right) T^{X}\left(\Lambda E^{*}, g^{\Lambda E^{*}}\right) \operatorname{ch}\left(\eta, g^{\eta}\right) \\
& \quad+\int_{Y} \operatorname{Td}{ }^{-1}\left(N_{Y / X}, g^{N_{Y / X}}\right) \widetilde{\operatorname{Td}}\left(T Y, T X_{\mid Y}, g^{T X_{\mid Y}}\right) \operatorname{ch}\left(\eta_{\mid Y}, g^{\eta_{\mid Y}}\right) \\
& \quad-\int_{X} \operatorname{Td}(T X) R(T X) \operatorname{ch}\left(\Lambda E^{*} \otimes \eta\right)+\int_{Y} \operatorname{Td}(T Y) R(T Y) \operatorname{ch}\left(\eta_{\mid Y}\right) .
\end{aligned}
$$

Remark 3.23. As the reader will have observed, the formal identities (3.69) have now become the rigorous identities (3.70). 
Also note that the result of Bismut-Lebeau [BL2] is valid for arbitrary resolutions of a complex vector bundle on $Y$ by a complex of holomorphic vector bundles on $X$, and not only for resolutions by Koszul complexes.

i) One Word of Explanation. As we just, saw, the result of Bismut-Lebeau [BL1,2] confirms the predictions of Theorem 3.20.

However the most essential point to observe is that the very structure of the rigorous proof of Theorem 3.22 in [BL2] is closely related to the proof of the result given in [B2, Theorem 3.4], with of course:

- many more technical difficulties,

- some essential differences.

The first basis result in [BL2] is as follows.

Theorem 3.24. Let $\tilde{\eta}_{u, T}$ be the 1-form on $R_{+}^{*} \times R_{+}^{*}$,

$$
\begin{aligned}
\tilde{\eta}_{u, T}= & \frac{d u}{u} \operatorname{Tr}_{s}\left[\left(N_{V}^{X}-\frac{\operatorname{dim} X}{2}\right) \exp \left(-\left(\sqrt{u} D^{X}+\sqrt{T} V\right)^{2}\right)\right] \\
& -\frac{d T}{T} \operatorname{Tr}_{s}\left[\left(N_{H}-\frac{\operatorname{dim} E}{2}\right) \exp \left(-\left(\sqrt{u} D^{X}+\sqrt{T} V\right)^{2}\right)\right]
\end{aligned}
$$

Then the form $\tilde{\eta}_{u, T}$ is closed.

Proof. Observe that by the McKean-Singer formula, $\operatorname{Tr}_{s}\left[\exp \left(-\left(\sqrt{u} D^{X}+\sqrt{T} V\right)^{2}\right)\right]$ is an integer which does not depend on $u$ or $T$. Our theorem then follows from [BL2, Theorem 3.3].

Remark 3.25. Recall that the form $\mu \in P^{L X}$ was defined in (3.48). Let $\eta_{u, T}$ be the one form on $R_{+}^{*} \times R_{+}^{*}$ which is defined formally by [B2, Eq. (3.12)], i.e. by an ill-defined integration process on the infinite dimensional manifold $L X$. In view of (3.9), (3.36)-(3.39), we get the formal equality

$$
\tilde{\eta}_{u, T}=\frac{1}{2 \pi i}\left(\frac{i C}{2 \pi}\right)^{\operatorname{dim} X-\operatorname{dim} E} \eta_{u, T}
$$

[B2, Theorem 3.6] and Theorem 3.24 are then formally equivalent. [B2, Theorem 3.6] plays a key role in the proof of [B2, Theorem 3.4]. Similarly, Theorem 3.24 is essential in the proof in [BL2] of Theorem 3.22.

Recall that $v=\sqrt{-1} i_{r}$. If the complex, $\left(\mathbb{K}, \bar{\partial}^{X}+v\right)$ has the properties described in Sect. 3d), the proof of Theorem 3.22 in Bismut-Lebeau [BL2] could be done by using the contour $\Gamma$ of [B2, Sect. 3c)] and by formally proceeding as in [B2]. However in general, these very special properties are not verified. This is why a change of variables $u \rightarrow u, T \rightarrow T u$ is done in [BL2, Theorem 3.5]. From the form $\tilde{\eta}_{u, T}$, we thus obtain a new closed form $\tilde{\lambda}_{u, T}$ on $R_{+}^{*} \times R_{+}^{*}$.

Let $\Gamma^{\prime}$ be the contour 


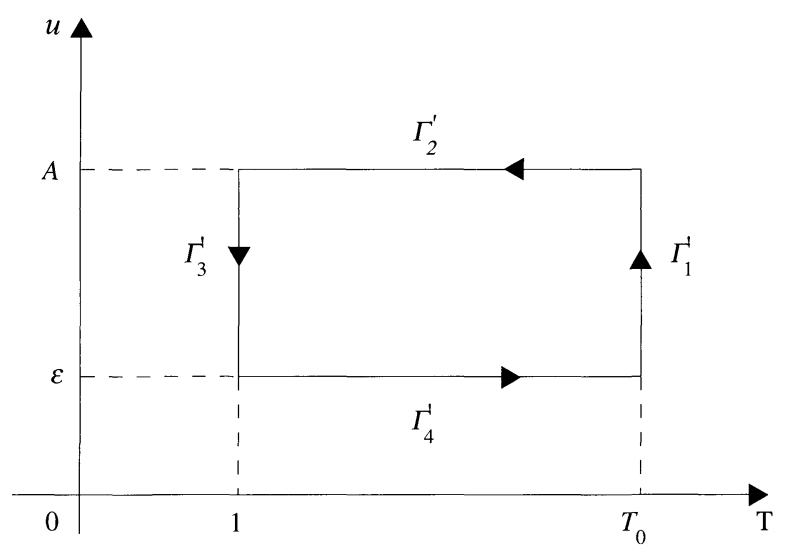

Theorem 3.22 is proved in [BL2] by starting from the identity

$$
\int_{\Gamma^{\prime}} \tilde{\lambda}=0
$$

and by making $A \rightarrow+\infty, T_{0} \rightarrow+\infty, \varepsilon \rightarrow 0$ in that order in (3.73).

Let $\bar{\partial}^{Y}$ be the Dolbeault operator acting on the $\mathbb{Z}$-graded vector space $\mathbb{L}$ of smooth sections of $\Lambda\left(T^{*(0,1)} Y\right) \otimes \eta_{\mid Y}$ over $Y$, and let $\bar{\partial}^{Y^{*}}$ be its formal adjoint. Set

$$
D^{Y}=\bar{\partial}^{Y}+\bar{\partial}^{Y^{*}}
$$

Let $N_{V}^{Y}$ be the number operator acting on $\mathbb{L}$. By Bismut-Lebeau [BL2, Theorem 6.4], we know that for any $u>0$,

$$
\begin{aligned}
& \lim _{T \rightarrow \infty} \operatorname{Tr}_{s}\left[\left(N_{V}^{X}-\frac{\operatorname{dim} X}{2}\right) \exp \left(-\left(\sqrt{u} D^{X}+\sqrt{T} V\right)^{2}\right)\right] \\
& \quad=\operatorname{Tr}_{s}\left[\left(N_{V}^{Y}-\frac{\operatorname{dim} Y}{2}\right) \exp \left(-u\left(D^{Y}\right)^{2}\right)\right], \\
& \lim _{T \rightarrow \infty} \operatorname{Tr}_{s}\left[\left(N_{H}-\frac{\operatorname{dim} E}{2}\right) \exp \left(-\left(\sqrt{u} D^{X}+\sqrt{T} V\right)^{2}\right)\right]=0 .
\end{aligned}
$$

The proofs of (3.75) rely on:

- A precise asymptotic analysis by an adequate rescaling of the operator $\sqrt{u} D^{X}+$ $\sqrt{T} V$ near $Y$ as $T \rightarrow+\infty$, which is done in [BL2, Sects. 8 and 9].

- A remarkable algebraic property of the kernel of certain harmonic oscillators acting on smooth form on $\mathbb{C}^{n}$, which is proved in [B7, Theorem 1.6]. This property explains in particular the second identity in (3.75). 
As explained in Remark 3.25, we have the formal identites

$$
\begin{aligned}
\operatorname{Tr}_{s} & {\left[\left(N_{V}^{X}-\frac{\operatorname{dim} X}{2}\right) \exp \left(-\left(\sqrt{u} D^{X}+\sqrt{T} V\right)^{2}\right)\right] } \\
= & \frac{1}{2 \pi i}\left(\frac{i C}{2 \pi}\right)^{\operatorname{dim} X-\operatorname{dim} E} \int_{L X} \mu^{K} \gamma_{u} s^{*} a_{T}\left(L E, g^{L E}\right) \\
\operatorname{Tr}_{s} & {\left[\left(N_{H}-\frac{\operatorname{dim} E}{2}\right) \exp \left(-\left(\sqrt{u} D^{X}+\sqrt{T} V\right)^{2}\right)\right] } \\
= & -\frac{1}{2 \pi i}\left(\frac{i C}{2 \pi}\right)^{\operatorname{dim} X-\operatorname{dim} E} \int_{L X} \mu^{K} \alpha_{u} s^{* K} c_{T}\left(L E, g^{L E}\right)
\end{aligned}
$$

By using [B2, Theorem 2.18] formally, we know that

$$
\begin{gathered}
\lim _{T \rightarrow \infty} \int_{L X} \mu^{K} \gamma_{u} s^{* K} a_{T}\left(L E, g^{L E}\right)=\int_{L Y} \mu^{K} \gamma_{u}, \\
\lim _{T \rightarrow \infty} \int_{L X} \mu^{K} \gamma_{u} s^{*} c_{T}\left(L E, g^{L E}\right)=0 .
\end{gathered}
$$

Therefore the formal formulas (3.76) exactly predict the correct answer in (3.75). Note that as appears clearly in [BGS5, Theorems 3.12 and 3.15], the second identity in (3.77) follows from the fact that

$$
\begin{aligned}
{ }^{K} c_{T}\left(L E, g^{L E}\right)= & \frac{\partial}{\partial b}\left[\operatorname{det}\left(-\frac{{ }^{K} R^{L E}}{2 i \pi}-b\right) \exp \left(-T \frac{|Z|^{2}}{2}\right)\right. \\
& \left.\sum_{k=0}^{\operatorname{dim} L E-1} \frac{\left(-T\left(^{K} R^{L E}+2 \pi b \mathbf{J}^{L E}\right)^{-1}\right)^{k}}{k !}\right]_{b=0},
\end{aligned}
$$

i.e. the expansion terminates at the index $k=\operatorname{dim} L E-1$ and not $\operatorname{dim} L E$.

Here $\operatorname{dim} L E=+\infty, \operatorname{dim} L E-1=+\infty$, and still by (3.75), the second identity in (3.77) is formally true!

In [BL2], the analysis of the terms $\int_{\Gamma_{1}^{\prime}} \tilde{\lambda}, \int_{\Gamma_{2}^{\prime}} \tilde{\lambda}$ relies on remarkable properties of the spectrum of the operator $D^{X}+T V$ as $T \rightarrow+\infty$, which are established in [BL2, Theorem 9.25]:

- The spectrum (with multiplicity) of $D^{X}+T V$ converges to the spectrum of $D^{Y}$. - For $T>0$, the dimension of the kernel of $D^{X}+T V$ is equal to the dimension of the kernel of $D^{Y}$.

If as in Sect.3d), we assume that $\operatorname{Ker} D^{Y}=\{0\}$, the analysis of the terms $I_{1}=$ $\int_{\Gamma_{1}^{\prime}} \tilde{\lambda}, I_{2}=\int_{\Gamma_{2}^{\prime}} \tilde{\lambda}$ is much simpler, and is closely related to the analysis of $I_{1}=\int_{\Gamma_{1}} \eta$, $I_{2}=\int_{\Gamma_{2}} \eta$ in $[\mathrm{B} 2$, Sect. 3e) $]$. The term $I_{3}=\int_{\Gamma_{3}^{\prime}} \tilde{\lambda}$ does not raise any special difficulty in [BL2]. 
In [BL'2] the analysis of $I_{4}=\int_{\Gamma_{4}^{\prime}} \tilde{\lambda}$ is very difficult, but is still very closely related to the analysis of $I_{4}=\int_{\Gamma_{4}} \eta$ in [B2, Sect. 3e)], especially at the last stage where $\varepsilon \rightarrow 0$. One essential difference is that, contrary to what happens in [B2], in [BL2], it is not easy to show that the problem is local near any arbitrary point $y \in Y$. To do this, Bismut and Lebeau [BL2, Sect. 13] use finite propagation speed for hyperbolic equations.

However as should be clear from [B7] and [BL2], the strict analogues of the forms $\chi_{T}$ which we also met in [B2, Sect. 1a)], appear when studying the limit as $\varepsilon \rightarrow 0$ of $I_{4}^{\prime}$. In [B7], the analogy is exhibited in the clearest way by a complete explicit calculation of certain infinite dimensional superconnection forms, which can be expressed as infinite products. Once these products are normalized (with infinite normalizing constant!), we obtain exactly the forms $\chi_{T}$.

Most of the arguments in the proofs of [B2] are also very useful in understanding the proof of Theorem 3.22 in Bismut-Lebeau [BL2] from an elementary point of view. An important difference should be pointed out here: the scalings are in general not exactly the same, in particular on the analogue of the coordinates $Z, \tilde{Z}$. Let us just say that in [BL2], $k_{u, T}$ which was defined in [B2, Eq. (3.94)] is replaced by $k_{u, T}^{\prime}$,

$$
k_{u, T}^{\prime}:\left(y, Z, Z^{\prime}, \tilde{Z}\right) \rightarrow\left(y,\left(\frac{u}{\sqrt{T}}\right)^{1 / 2} Z, \sqrt{u} Z^{\prime},\left(\frac{u}{\sqrt{T}}\right)^{1 / 2} \tilde{Z}\right)
$$

This change on the scaling reflects the infinite dimensional character of the problem considered in [BL2].

Acknowledgements. The author is indebted to a referee for his helfpul comments.

\section{References}

[A] Atiyah, M.F.: Circular symmetry and stationary phase approximation. Proceedings of the conference in honour of L. Schwartz. Astérisque 131, 43-59 (1985)

[AS] Atiyah, M.F., Singer, I.M.: The index of elliptic operators III. Ann. Math. 87, 546-604 (1968)

[BeV] Berline, N., Vergne, M.: Zéros d'un champ de vecteurs et classes caractéristiques équivariantes. Duke Math. J. 50, 539-549 (1983)

[B1] Bismut, J.-M.: Bott-Chern currents, excess normal bundles and the Chern character. To appear in G.A.F.A.

[B2] Bismut, J.-M.: Complex equivariant intersection, excess normal bundles and Bott-Chern currents. Commun. Math. Phys. 147, 1-55 (1992)

[B3] Bismut, J.-M.: Superconnection currents and complex immersions. Invent. Math. 99, 59-113 (1990)

[B4] Bismut, J.-M.: Index Theorem and equivariant cohomology on the Loop Space. Commun. Math. Phys. 98, 213-237 (1985)

[B5] Bismut, J.-M.: The Atiyah-Singer index Theorem for families of Dirac operators: Two heat equation proofs. Invent. Math. 83, 91-151 (1986)

[B6] Bismut, J.-M.: Localization formulas, superconnections and the index theorem for families. Commun. Math. Phys. 103, 127-166 (1986)

[B7] Bismut, J.-M.: Koszul complexes, harmonic oscillators and the Todd class. J.A.M.S. 3, 159-256 (1990)

[B8] Bismut, J.-M.: Equivariant Bott-Chern currents and the Ray-Singer analytic torsion. Math. Ann. 287, 495-507 (1990) 
[BGS1] Bismut, J.-M., Gillet, H., Soulé, C.: Analytic torsion and holomorphic determinant bundles. I. Commun. Math. Phys. 115, 49-78 (1988)

[BGS2] Bismut, J.-M., Gillet, H., Soulé, C.: Analytic torsion and holomorphic determinant bundles. II. Commun. Math. Phys. 115, 79-126 (1988)

[BGS3] Bismut, J.-M., Gillet, H., Soulé, C.: Analytic torsion and holomorphic determinant bundles. III. Commun. Math. Phys. 115, 301-351 (1988)

[BGS4] Bismut, J.-M., Gillet, H., Soulé, C.: Bott-Chern currents and complex immersions. Duke Math. J. 60, 255-284 (1990)

[BGS5] Bismut, J.-M., Gillet, H., Soulé, C.: Complex immersions and Arakelov geometry. The Grothendieck Festschrift, Cartier, P. et al. (eds.) pp. 249-331. Progress in Math. n 86. Boston, Basel, Berlin: Birkhäuser 1990

[BL1] Bismut, J.-M. Lebeau, G.: Immersions complexes et métriques de Quillen. C.R. Acad. Sci. Paris Sér. I. Math. 309, 487-491 (1989)

[BL2] Bismut, J.-M. Lebeau, G.: Complex immersions and Quillen metrics. Preprint Orsay 90-13 (1990). To appear in Publ. Math. IHES

[Bo] Bott, R.: Vector fields and characteristic numbers. Mich. Math. J. 14, 231-244 (1967)

[GS1] Gillet, H., Soulé, C.: Arithmetic Intersection Theory. Publ. Math. IHES, 72, 93-174 (1990)

[GS2] Gillet, H., Soulé, C.: Characteristic classes for algebraic vector bundles with Hermitian metric. Ann. Math. I, 131, 163-203 (1990), II, 131, 205-238 (1990)

[GS3] Gillet, H., Soulé, C.: Analytic torsion and the arithmetic Todd genus. Topology 30, 21-54 (1991)

[GS4] Gillet, H., Soulé, C.: Un théorème de Riemann-Roch-Grothendieck arithmétique. C.R. Acad. Sci Paris 309, Série I, 929-932 (1989)

[GS5] Gillet, H., Soulé, C.: An arithmetic Riemann-Roch theorem. Preprint IHES M/91/50

[H] Hitchin, N.J.: Harmonic spinors. Adv. Math. 14, 1-55 (1974)

[KMu] Knudsen, F.F., Mumford, D.: The projectivity of the moduli space of stable curves. I: Preliminaries on "det" and "div," Math. Scand. 39, 19-55 (1976)

[MQ] Mathai, V., Quillen, D.: Superconnections, Thom classes, and equivariant differential forms. Topology 25, 85-110 (1986)

[Q1] Quillen, D.: Superconnections and the Chern character. Topology 24, 89-95 (1985)

[Q2] Quillen, D.: Determinants of Cauchy-Riemann operators over a Riemann surface. Funct. Anal. Appl. 14, 31-34 (1985)

[RSi] Ray, D.B., Singer, I.M.: Analytic torsion for complex manifolds. Ann. Math. 98, 154-177 (1973)

[Se] Seeley, R.T.: Complex powers of an elliptic operator. Proc. Symp. Pure Appl. Math. AMS 10, 288-307 (1967)

Communicated by A. Jaffe 